\title{
A WEAK GALERKIN MIXED FINITE ELEMENT METHOD FOR SECOND ORDER ELLIPTIC PROBLEMS
}

\author{
JUNPING WANG AND XIU YE
}

\begin{abstract}
A new weak Galerkin (WG) method is introduced and analyzed for the second order elliptic equation formulated as a system of two first order linear equations. This method, called WG-MFEM, is designed by using discontinuous piecewise polynomials on finite element partitions with arbitrary shape of polygons/polyhedra. The WG-MFEM is capable of providing very accurate numerical approximations for both the primary and flux variables. Allowing the use of discontinuous approximating functions on arbitrary shape of polygons/polyhedra makes the method highly flexible in practical computation. Optimal order error estimates in both discrete $H^{1}$ and $L^{2}$ norms are established for the corresponding weak Galerkin mixed finite element solutions.
\end{abstract}

\section{INTRODUCTION}

Weak Galerkin (WG) refers to a finite element technique for partial differential equations in which differential operators are approximated by their weak forms as distributions. In 29,30, a weak Galerkin method was introduced and analyzed for second order elliptic equations and the Stokes equation based on weak gradients and weak divergence. In this paper, we shall develop a new weak Galerkin method for second order elliptic equations formulated as a system of two first order linear equations. Our model problem seeks a flux function $\mathbf{q}=\mathbf{q}(\mathbf{x})$ and a scalar function $u=u(\mathbf{x})$ defined in an open bounded polygonal or polyhedral domain $\Omega \subset \mathbb{R}^{d}(d=$ $2,3)$ satisfying

$$
\alpha \mathbf{q}+\nabla u=0, \nabla \cdot \mathbf{q}=f, \quad \text { in } \Omega
$$

and the following Dirichlet boundary condition

$$
u=-g \text { on } \partial \Omega,
$$

where $\alpha=\left(\alpha_{i j}(\mathbf{x})\right)_{d \times d} \in\left[L^{\infty}(\Omega)\right]^{d^{2}}$ is a symmetric, uniformly positive definite matrix on the domain $\Omega$. A weak formulation for (1.1)-(1.2) seeks $\mathbf{q} \in H(\operatorname{div}, \Omega)$

Received by the editor March 20, 2012 and, in revised form, November 23, 2012 and December $11,2012$.

2010 Mathematics Subject Classification. Primary, 65N15, 65N30, 76D07; Secondary, 35B45, $35 \mathrm{~J} 50$.

Key words and phrases. Weak Galerkin, finite element methods, discrete weak divergence, second order elliptic problems, mixed finite element methods.

The research of the first author was supported by the NSF IR/D program, while working at the Foundation. However, any opinion, finding, and conclusions or recommendations expressed in this material are those of the author and do not necessarily reflect the views of the National Science Foundation.

This research was supported in part by National Science Foundation Grant DMS-1115097. 
and $u \in L^{2}(\Omega)$ such that

$$
\begin{aligned}
(\alpha \mathbf{q}, \mathbf{v})-(\nabla \cdot \mathbf{v}, u) & =\langle g, \mathbf{v} \cdot \mathbf{n}\rangle_{\partial \Omega}, \quad \forall \mathbf{v} \in H(\operatorname{div}, \Omega) \\
(\nabla \cdot \mathbf{q}, w) & =(f, w), \quad \forall w \in L^{2}(\Omega) .
\end{aligned}
$$

Here $L^{2}(\Omega)$ is the standard Hilbert space of square integrable functions on $\Omega, \nabla \cdot \mathbf{v}$ is the divergence of vector-valued functions $\mathbf{v}$ on $\Omega, H(d i v, \Omega)$ is the Sobolev space consisting of vector-valued functions $\mathbf{v}$ such that $\mathbf{v} \in\left[L^{2}(\Omega)\right]^{d}$ and $\nabla \cdot \mathbf{v} \in L^{2}(\Omega)$, $(\cdot, \cdot)$ stands for the $L^{2}$-inner product in $L^{2}(\Omega)$, and $\langle\cdot, \cdot\rangle_{\partial \Omega}$ is the inner product in $L^{2}(\partial \Omega)$.

Galerkin methods based on the weak formulation (1.3)-(1.4) and finite dimensional subspaces of $H($ div,$\Omega) \times L^{2}(\Omega)$ with piecewise polynomials are known as mixed finite element methods (MFEM). MFEMs for (1.1)-(1.2) treat $\mathbf{q}$ and $u$ as unknown functions and are capable of providing accurate approximations for both unknowns [1, 3, 7-11, 27, 28. All the existing MFEMs in literature possess local mass conservation that makes MFEM a competitive numerical technique in many applications such as oil reservoir and groundwater flow simulation in porous media. On the other hand, MFEMs are formulated in subspaces of $H(\operatorname{div}, \Omega) \times L^{2}(\Omega)$ which requires a certain continuity of the finite element functions for the flux variable. More precisely, the flux functions must be sufficiently continuous so that the usual divergence is well-defined in the classical sense in $L^{2}(\Omega)$. This continuity assumption in turn imposes a strong restriction on the structure of the finite element partition and the piecewise polynomials defined on them [9 11,27, 30.

The weak Galerkin method introduced in [29] (see also [22, 23] for extensions) was based on the use of weak gradients in the following variational formulation: find $u \in H^{1}(\Omega)$ such that $u=-g$ on $\partial \Omega$ and

$$
\left(\alpha^{-1} \nabla u, \nabla \phi\right)=(f, \phi), \quad \forall \phi \in H_{0}^{1}(\Omega),
$$

where $H^{1}(\Omega)$ is the Sobolev space consisting of functions for which all partial derivatives up to order one are square integrable, $H_{0}^{1}(\Omega)$ is the subspace of $H^{1}(\Omega)$ consisting of functions with vanishing value on $\partial \Omega$. Specifically, the weak Galerkin finite element formulation in $22,23,29,30$ can be obtained from (1.5) by simply replacing the gradient $\nabla$ by a discrete gradient $\nabla_{w}$ (it was denoted as $\nabla_{d}$ in [29]) defined by a distributional formula. The discrete weak gradient operator $\nabla_{w}$ is locally-defined on each element. It has been demonstrated [24, 26, 29, 30] that the weak Galerkin method enjoys an easy-to-implement formulation that is parameter free and inherits the physical property of mass conservation locally on each element. Furthermore, the weak Galerkin method has the flexibility of using discontinuous finite element functions, as was commonly employed in discontinuous Galerkin and hybridized discontinuous Galerkin methods [2,15.

The goal of this paper is to extend the weak Galerkin method of [22,23, 29] to the variational formulation (1.3)-(1.4) by following the idea of weak gradients. It is clear that divergence is the principle differential operator in (1.3)-(1.4). Thus, an essential part of the extension is the development of a weakly-defined discrete divergence operator, denoted by $\left(\nabla_{w} \cdot\right)$, for a class of vector-valued weak functions in a finite element setting. Assuming that there is such a discrete divergence operator $\left(\nabla_{w} \cdot\right)$ defined on a finite element space $\mathcal{V}_{h}$ for the flux variable $\mathbf{q}$, then formally one would have a WG method for (1.1)-(1.2) that seeks $\mathbf{q}_{h} \in \mathcal{V}_{h}$ and $u_{h} \in \mathcal{W}_{h}$ 
satisfying

$$
\begin{aligned}
\left(\alpha \mathbf{q}_{h}, \mathbf{v}\right)-\left(\nabla_{w} \cdot \mathbf{v}, u_{h}\right) & =\langle g, \mathbf{v} \cdot \mathbf{n}\rangle_{\partial \Omega}, \quad \forall \mathbf{v} \in \mathcal{V}_{h} \\
\left(\nabla_{w} \cdot \mathbf{q}_{h}, w\right) & =(f, w), \quad \forall w \in \mathcal{W}_{h},
\end{aligned}
$$

where $\mathcal{W}_{h}$ is a properly defined finite element space for the scalar variable. The rest of the paper will provide details for a rigorous interpretation and justification of the above formal WG method, which shall be called WG-MFEM methods.

The WG-MFEM have the following features. First of all, the finite element partition of the domain $\Omega$ is allowed to consist of arbitrary shape of polygons for $d=2$ and polyhedra for $d=3$. Second, the flux approximation space $\mathcal{V}_{h}$ consists of two components, where the first one is given by piecewise polynomials on each polygon/polyhedra and the second is given by piecewise polynomials on the edges/faces of the polygon/polyhedra. The second component shall be used to approximate the normal component of the flux variable $\mathbf{q}$ on each edge/face. Furthermore, the scalar approximation space $\mathcal{W}_{h}$ consists of piecewise polynomials on each polygon/polyhedra with one degree higher than that of the flux. For example, the lowest order of such elements would consist of piecewise constant for flux and its normal component on each edge/face plus piecewise linear function for the scalar variable on each polygon/polyhedra. There is no continuity required for any of the finite element functions in WG-MFEM.

One close relative of the WG-MFEM is the mimetic finite difference (MFD) method, see [4, 6, 12 and the references cited therein. Both WG-MFEM and MFD share the same flexibility of using polygonal/polyhedral elements of the domain. The lowest order MFD approximates the flux by using only piecewise constants on each edge/face, and the scalar variable by using another piecewise constant function on each polygonal/polyhedral element, while WG-MFEM provides a wide class of numerical schemes with arbitrary order of polynomials. The arbitrary-order mimetic scheme of [4 is based on the philosophy of approximating the forms in (1.5) using nodal-based polynomials, which has very minimal overlap with the WGMFEM. It should be pointed out that there are other numerical methods designed on general polygonal meshes in existing literature [2, 15, 17, 20]. In particular, a comparison with existing numerical schemes should be conducted on a certain set of benchmark problems as demonstrated in [20]. More benchmarks can be found in 19 .

Allowing arbitrary shape for mesh elements provides a convenient flexibility in both numerical approximation and mesh generation, especially in regions where the domain geometry is complex. Such a flexibility is also very much appreciated in adaptive mesh refinement methods. This is a highly desirable feature in practice since a single type of mesh technology is too restrictive in resolving complex multidimensional and multi-scale problems efficiently [21.

This paper is organized as follows. In Section 2, we present a discussion of weak divergence operator in some weakly-defined spaces. In Section 3, we provide a detailed description and assumptions for the WG-MFEM. In Section 4, we define some local projection operators and then derive some approximation properties which are useful in error analysis. In Section 5, we shall establish an optimal order error estimate for the WG-MFEM approximations in a norm that is related to the $L^{2}$ for the flux and $H^{1}$ for the scalar function. In Section [6, we derive an optimal order error estimate in $L^{2}$ for the scalar approximation by using a duality argument 
as was commonly employed in the standard Galerkin finite element methods [7, 14]. Finally, we provide some technical results in the appendix that are critical in dealing with finite element functions on arbitrary polygons/polyhedra.

\section{WEAK DIVERGENCE}

The key in weak Galerkin methods is the use of weak derivatives in the place of strong derivatives in the variational form for the underlying partial differential equations. For the mixed problem (1.1) with boundary condition (1.2), the corresponding variational form is given by (1.3) and (1.4), where divergence is the only differential operator involved in the formulation. Thus, understanding weak divergence is critically important in the corresponding WG method. The goal of this section is to introduce a weak divergence operator and its approximation by using piecewise polynomials.

Let $K$ be any polygonal or polyhedral domain with boundary $\partial K$. A weak vectorvalued function on the region $K$ refers to a vector-valued function $\mathbf{v}=\left\{\mathbf{v}_{0}, \mathbf{v}_{b}\right\}$ such that $\mathbf{v}_{0} \in\left[L^{2}(K)\right]^{d}$ and $\mathbf{v}_{b} \cdot \mathbf{n} \in H^{-\frac{1}{2}}(\partial K)$, where $\mathbf{n}$ is the outward normal direction of $K$ on its boundary. The first component $\mathbf{v}_{0}$ can be understood as the value of $\mathbf{v}$ in the interior of $K$, and the second component $\mathbf{v}_{b}$ represents $\mathbf{v}$ on the boundary of $K$. The requirement of $\mathbf{v}_{b} \cdot \mathbf{n} \in H^{-\frac{1}{2}}(\partial K)$ indicates that we are merely interested in the normal component of $\mathbf{v}_{b}$. Note that $\mathbf{v}_{b}$ may not be necessarily related to the trace of $\mathbf{v}_{0}$ on $\partial K$ should a trace be well defined. Denote by $\mathcal{M}(K)$ the space of weak vector-valued functions on $K$; i.e.,

$$
\mathcal{M}(K)=\left\{\mathbf{v}=\left\{\mathbf{v}_{0}, \mathbf{v}_{b}\right\}: \mathbf{v}_{0} \in\left[L^{2}(K)\right]^{d}, \mathbf{v}_{b} \cdot \mathbf{n} \in H^{-\frac{1}{2}}(\partial K)\right\} .
$$

Following the definition of weak gradient introduced in [29], we define a weak divergence operator as follows.

Definition 2.1. The dual of $L^{2}(K)$ can be identified with itself by using the standard $L^{2}$ inner product as the action of linear functionals. With a similar interpretation, for any $\mathbf{v} \in \mathcal{M}(K)$, the weak divergence of $\mathbf{v}$ is defined as a linear functional $\nabla_{w} \cdot \mathbf{v}$ in the dual space of $H^{1}(K)$ whose action on each $\varphi \in H^{1}(K)$ is given by

$$
\left(\nabla_{w} \cdot \mathbf{v}, \varphi\right)_{K}:=-\left(\mathbf{v}_{0}, \nabla \varphi\right)_{K}+\left\langle\mathbf{v}_{b} \cdot \mathbf{n}, \varphi\right\rangle_{\partial K},
$$

where $\mathbf{n}$ is the outward normal direction to $\partial K,\left(\mathbf{v}_{0}, \nabla \varphi\right)_{K}=\int_{K} \mathbf{v}_{0} \cdot \nabla \varphi d K$ is the action of $\mathbf{v}_{0}$ on $\nabla \varphi$, and $\left\langle\mathbf{v}_{b} \cdot \mathbf{n}, \varphi\right\rangle_{\partial K}$ is the action of $\mathbf{v}_{b} \cdot \mathbf{n}$ on $\varphi \in H^{\frac{1}{2}}(\partial K)$.

The Sobolev space $\left[H^{1}(K)\right]^{d}$ can be embedded into the space $\mathcal{M}(K)$ by an inclusion map $i_{\mathcal{M}}:\left[H^{1}(K)\right]^{d} \rightarrow \mathcal{M}(K)$ defined as follows:

$$
i_{\mathcal{M}}(\mathbf{q})=\left\{\left.\mathbf{q}\right|_{K},\left.\mathbf{q}\right|_{\partial K}\right\} .
$$

With the help of the inclusion map $i_{\mathcal{M}}$, the Sobolev space $\left[H^{1}(K)\right]^{d}$ can be viewed as a subspace of $\mathcal{M}(K)$ by identifying each $\mathbf{q} \in\left[H^{1}(K)\right]^{d}$ with $i_{\mathcal{M}}(\mathbf{q})$. Analogously, a weak vector-valued function $\mathbf{v}=\left\{\mathbf{v}_{0}, \mathbf{v}_{b}\right\} \in \mathcal{M}(K)$ is said to be in $\left[H^{1}(K)\right]^{d}$ if it can be identified with a function $\mathbf{q} \in\left[H^{1}(K)\right]^{d}$ through the above inclusion map. It is not hard to see that $\nabla_{w} \cdot \mathbf{v}=\nabla \cdot \mathbf{v}$ if $\mathbf{v}$ is a smooth function in $\left[H^{1}(K)\right]^{d}$.

Next, we introduce a discrete weak divergence operator by approximating $\left(\nabla_{w} \cdot\right)$ in a polynomial subspace of the dual of $H^{1}(K)$. To this end, for any non-negative integer $r \geq 0$, denote by $P_{r}(K)$ the set of polynomials on $K$ with degree no more 


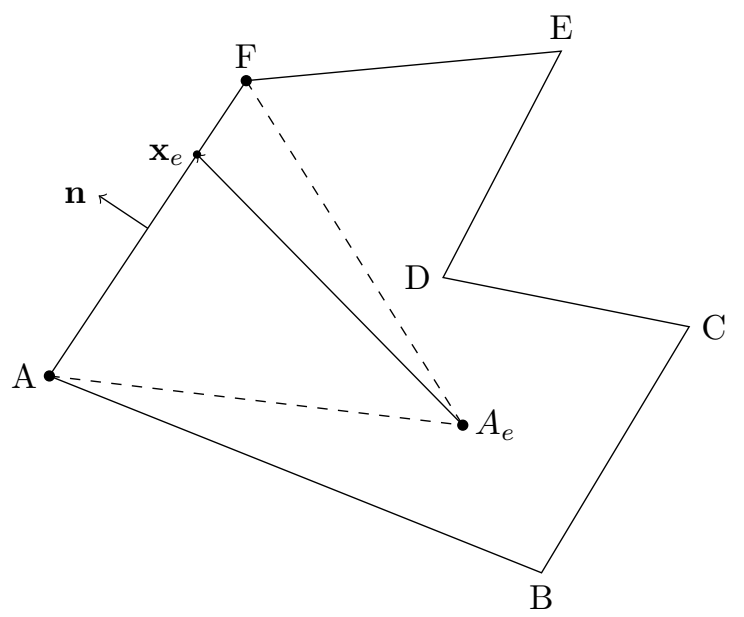

FiguRE 1. Depiction of a shape-regular polygonal element $A B C D E F A$.

than $r$. A discrete weak divergence operator, denoted by $\left(\nabla_{w, r} \cdot\right)$, is defined as the unique polynomial $\left(\nabla_{w, r} \cdot \mathbf{v}\right) \in P_{r}(K)$ that satisfies the following equation:

$$
\left(\nabla_{w, r} \cdot \mathbf{v}, \phi\right)_{K}=-\left(\mathbf{v}_{0}, \nabla \phi\right)_{K}+\left\langle\mathbf{v}_{b} \cdot \mathbf{n}, \phi\right\rangle_{\partial K}, \quad \forall \phi \in P_{r}(K) .
$$

\section{Weak Galerkin MFEM: Assumptions and Algorithm}

Let $\mathcal{T}_{h}$ be a partition of the domain $\Omega$ consisting of polygons in $2 \mathrm{D}$ and polyhedra in $3 \mathrm{D}$. Denote by $\mathcal{E}_{h}$ the set of all edges or flat faces in $\mathcal{T}_{h}$, and let $\mathcal{E}_{h}^{0}=\mathcal{E}_{h} \backslash \partial \Omega$ be the set of all interior edges or flat faces. For every element $T \in \mathcal{T}_{h}$, we denote by $|T|$ the area or volume of $T$ and by $h_{T}$ its diameter. Similarly, we denote by $|e|$ the length or area of $e$ and by $h_{e}$ the diameter of edge or flat face $e \in \mathcal{E}_{h}$. We also set as usual the mesh size of $\mathcal{T}_{h}$ by

$$
h=\max _{T \in \mathcal{T}_{h}} h_{T} .
$$

All the elements of $\mathcal{T}_{h}$ are assumed to be closed and simply connected polygons or polyhedra; see Figure 1. We need some shape regularity for the partition $\mathcal{T}_{h}$ described as below.

A1: Assume that there exist two positive constants $\varrho_{v}$ and $\varrho_{e}$ such that for every element $T \in \mathcal{T}_{h}$ we have

$$
\varrho_{v} h_{T}^{d} \leq|T|, \quad \varrho_{e} h_{e}^{d-1} \leq|e|
$$

for all edges or flat faces of $T$.

A2: Assume that there exists a positive constant $\kappa$ such that for every element $T \in \mathcal{T}_{h}$ we have

$$
\kappa h_{T} \leq h_{e}
$$

for all edges or flat faces $e$ of $T$.

A3: Assume that the mesh edges or faces are flat. We further assume that for every $T \in \mathcal{T}_{h}$, and for every edge/face $e \in \partial T$, there exists a pyramid $P\left(e, T, A_{e}\right)$ contained in $T$ such that its base is identical with $e$, its apex is $A_{e} \in T$, and its height is proportional to $h_{T}$ with a proportionality constant 
$\sigma_{e}$ bounded away from a fixed positive number $\sigma^{*}$ from below. In other words, the height of the pyramid is given by $\sigma_{e} h_{T}$ such that $\sigma_{e} \geq \sigma^{*}>0$. The pyramid is also assumed to stand up above the base $e$ in the sense that the angle between the vector $\mathbf{x}_{e}-A_{e}$, for any $x_{e} \in e$, and the outward normal direction of $e$ (i.e., the vector $\mathbf{n}$ in Figure 10 is strictly acute by falling into an interval $\left[0, \theta_{0}\right]$ with $\theta_{0}<\frac{\pi}{2}$.

A4: Assume that each $T \in \mathcal{T}_{h}$ has a circumscribed simplex $S(T)$ that is shape regular and has a diameter $h_{S(T)}$ proportional to the diameter of $T$; i.e., $h_{S(T)} \leq \gamma_{*} h_{T}$ with a constant $\gamma_{*}$ independent of $T$. Furthermore, assume that each circumscribed simplex $S(T)$ intersects with only a fixed and small number of such simplices for all other elements $T \in \mathcal{T}_{h}$.

Figure 1 depicts a polygonal element that is shape regular. Readers are referred to 12 for a similar, but different type of shape regularity assumption for the underlying finite element partition of the domain. The shape regularity is required for deriving error estimates for locally defined projection operators to be detailed in later sections.

Recall that, on each element $T \in \mathcal{T}_{h}$, there is a space of weak vector-valued functions $\mathcal{M}(T)$ defined as in (6.11). Denote by $\mathcal{M}$ the weak vector-valued function space on $\mathcal{T}_{h}$ given by

$$
\mathcal{M}:=\prod_{T \in \mathcal{T}_{h}} \mathcal{M}(T)
$$

Note that functions in $\mathcal{M}$ are defined on each element, and there are two-sided values of $\mathbf{v}_{b}$ on each interior edge/face $e$, depicted as $\left.\mathbf{v}_{b}\right|_{\partial T_{1}}$ and $\left.\mathbf{v}_{b}\right|_{\partial T_{2}}$ in Figure 2,

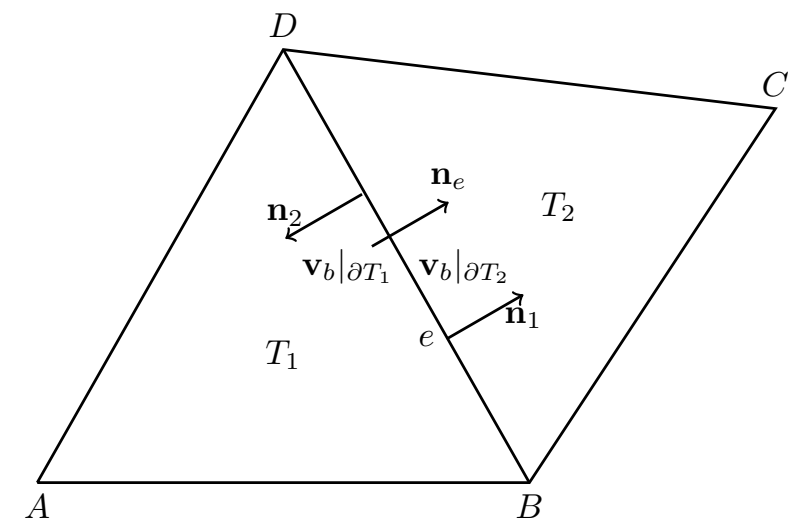

Figure 2. An interior edge shared by two elements

Let $\mathcal{V} \subset \mathcal{M}$ be a subspace of $\mathcal{M}$ consisting of weak vector-valued functions which are continuous across each interior edge/face $e$ in the normal direction; i.e.,

$$
\mathcal{V}=\left\{\mathbf{v} \in \mathcal{M}: \exists \mathbf{q} \in H(\operatorname{div}, \Omega) \text { s.t. }\left.\mathbf{q}\right|_{\partial T} \cdot \mathbf{n}=\left(\mathbf{v}_{b}\right)_{\partial T} \cdot \mathbf{n}, \forall T \in \mathcal{T}_{h}\right\} .
$$

The weak divergence operator as defined in (2.2) can be extended to any weak vector-valued function $\mathbf{v} \in \mathcal{V}$ by taking weak divergence locally on each element $T$. 
More precisely, the weak divergence of any $\mathbf{v} \in \mathcal{V}$ is defined element-by-element as follows:

$$
\nabla_{w} \cdot \mathbf{v}=\nabla_{w} \cdot\left(\left.\mathbf{v}\right|_{T}\right), \quad \text { on } T \in \mathcal{T}_{h} .
$$

Similarly, the discrete weak divergence as defined in (2.3) can be extended to $\mathcal{V}$ by defining

$$
\nabla_{w, r} \cdot \mathbf{v}=\nabla_{w, r} \cdot\left(\left.\mathbf{v}\right|_{T}\right), \quad \text { on } T \in \mathcal{T}_{h} .
$$

The definition of weak divergence of $\mathbf{v}=\left\{\mathbf{v}_{0}, \mathbf{v}_{b}\right\} \in \mathcal{V}$ requires the value of $\mathbf{v}$ on each element $T$, namely $\mathbf{v}_{0}$, and the normal component of $\mathbf{v}_{b}$ on each edge or face $e \in \mathcal{E}_{h}$. Thus, it is the normal component of $\mathbf{v}$ on each $e \in \mathcal{E}_{h}$ that really enters into the equation of discussion in numerical methods. For convenience, we introduce a set of normal directions on $\mathcal{E}_{h}$ as follows:

$$
\mathcal{D}_{h}=\left\{\mathbf{n}_{e}: \mathbf{n}_{e} \text { is unit and normal to } e, e \in \mathcal{E}_{h}\right\} .
$$

Figure 2 shows one example of $\mathbf{n}_{e}$ pointing from the element $T_{1}$ to $T_{2}$. In the rest of this paper, we will be concerned with a subspace of $\mathcal{V}$ in which the second component of $\mathbf{v}=\left\{\mathbf{v}_{0}, \mathbf{v}_{b}\right\}$ represents the normal component of $\mathbf{v}$ on each $e \in \mathcal{E}_{h}$; i.e., $\left.\left(\mathbf{v}_{b}\right)\right|_{e}=\left(\left.\mathbf{v}\right|_{e} \cdot \mathbf{n}_{e}\right) \mathbf{n}_{e}$.

A discrete weak vector-valued function $\mathbf{v}=\left\{\mathbf{v}_{0}, \mathbf{v}_{b}\right\}$ refers to weak vector-valued functions in which both $\mathbf{v}_{0}$ and $\mathbf{v}_{b}$ are vector-valued polynomials. Since the second component is represented as $\mathbf{v}_{b}=v_{b} \mathbf{n}_{e}$ where $\mathbf{n}_{e}$ is the prescribed normal direction to $e \in \mathcal{E}_{h}$, then $v_{b}$ is required to be a polynomial on each edge or flat face of $\partial T$. Recall that, for each element $T \in \mathcal{T}_{h}, P_{k}(T)$ denotes the set of polynomials on $T$ with degree no more than $k \geq 0$ and $P_{\ell}(e)$ is the set of polynomials on $e \in \mathcal{E}_{h}$ with degree no more than $\ell \geq 0$.

We now introduce two finite element spaces which are necessary for formulating our numerical schemes. The first one corresponds to the scalar (or pressure) variable defined as

$$
\mathcal{W}_{h}=\left\{w \in L^{2}(\Omega) ;\left.w\right|_{T} \in P_{k+1}(T)\right\},
$$

where $k \geq 0$ is a non-negative integer. The second one corresponds to vector-valued functions and their normal components on the set $\mathcal{E}_{h}$ of edges or flat faces, and is given by

$$
\mathcal{V}_{h}=\left\{\mathbf{v}=\left\{\mathbf{v}_{0}, \mathbf{v}_{b}\right\}:\left.\mathbf{v}_{0}\right|_{T} \in\left[P_{k}(T)\right]^{d},\left.\mathbf{v}_{b}\right|_{e}=v_{b} \mathbf{n}_{e}, v_{b} \in P_{k}(e), e \in \mathcal{E}_{h}\right\} .
$$

The pair $\mathcal{V}_{h} \times \mathcal{W}_{h}$ forms a finite element approximation space for the unknowns $\mathbf{q}$ and $u$ of the problem (1.3)-(1.4). For simplicity of notation and discussion, we shall refer to the above defined finite element spaces as the $\left(\left[P_{k}(T)\right]^{d}, P_{k}(e), P_{k+1}(T)\right)$ elements. The lowest order of such elements makes use of piecewise constant for the flux variable on each element $T$ and its edges/faces and piecewise linear for the pressure (scalar) variable.

The discrete weak divergence $\left(\nabla_{w, k+1} \cdot\right)$ as defined in (3.5) and (2.3) then provides a linear map from the finite element space $\mathcal{V}_{h}$ to $\mathcal{W}_{h}$. In particular, for any $\mathbf{v} \in \mathcal{V}_{h}$ and $w \in \mathcal{W}_{h}$, we have the following relation:

$$
\begin{aligned}
\left(\nabla_{w, k+1} \cdot \mathbf{v}, w\right): & =\sum_{T \in \mathcal{T}_{h}}\left(\nabla_{w, k+1} \cdot \mathbf{v}, w\right)_{T} \\
& =\sum_{T \in \mathcal{T}_{h}}\left(\int_{\partial T}\left(\mathbf{v}_{b} \cdot \mathbf{n}\right) w d s-\int_{T} \mathbf{v}_{0} \cdot(\nabla w) d T\right)
\end{aligned}
$$


With an abuse of notation, we shall use $\left(\nabla_{w} \cdot\right)$ to denote the discrete weak divergence operator $\left(\nabla_{w, k+1} \cdot\right)$ in the rest of this paper.

With the discrete divergence given by (3.8), one might naively formulate a finite element method by using (1.3) and (1.4) as follows. Find $\mathbf{q}_{h}=\left\{\mathbf{q}_{0}, \mathbf{q}_{b}\right\} \in \mathcal{V}_{h}$ and $u_{h} \in \mathcal{W}_{h}$ such that

$$
\begin{aligned}
\left(\alpha \mathbf{q}_{0}, \mathbf{v}_{0}\right)-\left(\nabla_{w} \cdot \mathbf{v}, u_{h}\right) & =\left\langle g, \mathbf{v}_{b} \cdot \mathbf{n}\right\rangle_{\partial \Omega}, \quad \forall \mathbf{v}=\left\{\mathbf{v}_{0}, \mathbf{v}_{b}\right\} \in \mathcal{V}_{h}, \\
\left(\nabla_{w} \cdot \mathbf{q}_{h}, w\right) & =(f, w), \quad \forall w \in \mathcal{W}_{h} .
\end{aligned}
$$

Unfortunately, due to an insufficient enforcement on the component $\mathbf{q}_{b}$, the resulting system of linear equations from (3.9)-3.10) generally does not have a unique solution. One remedy to this problem is to stabilize the bilinear form $\left(\alpha \mathbf{q}_{0}, \mathbf{v}_{0}\right)$ by requiring some communication between $\mathbf{q}_{0}$ and $\mathbf{q}_{b}$. To this end, we introduce three bilinear forms as follows.

$$
\begin{aligned}
& a(\boldsymbol{\eta}, \mathbf{v})=\left(\alpha \boldsymbol{\eta}_{0}, \mathbf{v}_{0}\right), \quad \boldsymbol{\eta}, \mathbf{v} \in \mathcal{V}_{h} \\
& b(\boldsymbol{\eta}, w)=\left(\nabla_{w} \cdot \boldsymbol{\eta}, w\right), \quad \boldsymbol{\eta} \in \mathcal{V}_{h}, w \in \mathcal{W}_{h}, \\
& s(\boldsymbol{\eta}, \mathbf{v})=\rho \sum_{T \in \mathcal{T}_{h}} h_{T}\left\langle\left(\boldsymbol{\eta}_{0}-\boldsymbol{\eta}_{b}\right) \cdot \mathbf{n},\left(\mathbf{v}_{0}-\mathbf{v}_{b}\right) \cdot \mathbf{n}\right\rangle_{\partial T}, \quad \boldsymbol{\eta}, \mathbf{v} \in \mathcal{V}_{h}
\end{aligned}
$$

and a stabilized bilinear form $a_{s}(\cdot, \cdot)$ :

$$
a_{s}(\boldsymbol{\eta}, \mathbf{v}):=a(\boldsymbol{\eta}, \mathbf{v})+s(\boldsymbol{\eta}, \mathbf{v}) .
$$

Here $\rho>0$ is any parameter and $h_{T}$ is the size of $T$. In practical computation, one might chose $\rho=1$ and substitute $h_{T}$ by the mesh size $h$ for quasi-uniform partitions; i.e., partitions for which $h_{T} / h$ is bounded from below and above uniformly in $T$.

Weak Galerkin MFEM Algorithm. Let $\mathcal{T}_{h}$ be a shape regular finite element partition of $\Omega$ and $\mathcal{V}_{h} \times \mathcal{W}_{h}$ be the corresponding finite element spaces consisting of $\left(\left[P_{k}(T)\right]^{d}, P_{k}(e), P_{k+1}(T)\right)$ elements with $k \geq 0$. An approximation for (1.1) -(1.2) is given by seeking $\mathbf{q}_{h}=\left\{\mathbf{q}_{0}, \mathbf{q}_{b}\right\} \in \mathcal{V}_{h}$ and $u_{h} \in \mathcal{W}_{h}$ such that

$$
\begin{aligned}
a_{s}\left(\mathbf{q}_{h}, \mathbf{v}\right)-b\left(\mathbf{v}, u_{h}\right) & =\left\langle g, \mathbf{v}_{b} \cdot \mathbf{n}\right\rangle_{\partial \Omega}, \\
b\left(\mathbf{q}_{h}, w\right) & =(f, w),
\end{aligned}
$$

for all $\mathbf{v}=\left\{\mathbf{v}_{0}, \mathbf{v}_{b}\right\} \in \mathcal{V}_{h}$ and $w \in \mathcal{W}_{h}$. The pair of solutions $\left(\mathbf{q}_{h} ; u_{h}\right)$ is called $a$ weak Galerkin mixed finite element approximation of (1.1)-(1.2).

The WG-MFEM scheme (3.14)-(3.15) retains the mass conservation property of (1.1)-(1.2) locally on each element. This can be seen by choosing a piecewise constant test function $w$ in (3.15). More precisely, for any element $T \in \mathcal{T}_{h}$, let $w$ assume the value 1 on $T$ and zero elsewhere. It follows from (3.15) that

$$
\left(\nabla_{w} \cdot \mathbf{q}_{h}, 1\right)_{T}=(f, 1)_{T} .
$$

The definition of discrete weak divergence (3.8) then implies the identity

$$
\int_{\partial T} \mathbf{q}_{b} \cdot \mathbf{n} d s=\int_{T} f d x
$$

which asserts a mass conservation for the WG-MFEM method.

The rest of the paper shall provide a theoretical foundation for the solvability and accuracy of the weak Galerkin mixed finite element scheme (3.14)-(3.15). 


\section{Local Projections: Definition and Properties}

We introduce two projection operators into the finite element spaces $\mathcal{V}_{h}$ and $\mathcal{W}_{h}$ by using local $L^{2}$ projections. To this end, for each element $T \in \mathcal{T}_{h}$, denote by $Q_{0}$ the usual $L^{2}$ projection from $L^{2}(T)$ onto $P_{k}(T)$. Similarly, for each edge or flat face $e \in \mathcal{E}_{h}$, let $Q_{b}$ be the $L^{2}$ projection from $L^{2}(e)$ onto $P_{k}(e)$. For any $\mathbf{v}=\left\{\mathbf{v}_{0}, v_{b} \mathbf{n}_{e}\right\} \in \mathcal{V}$ with $v_{b} \in L^{2}(e)$ on each edge/face $e$, denote by

$$
Q_{h} \mathbf{v}:=\left\{Q_{0}\left(\mathbf{v}_{0}\right), Q_{b}\left(v_{b}\right) \mathbf{n}_{e}\right\}
$$

the $L^{2}$ projection of $\mathbf{v}$ in $\mathcal{V}_{h}$. Next, denote by $\mathbb{Q}_{h}$ the $L^{2}$ projection from $L^{2}(\Omega)$ onto $\mathcal{W}_{h}$. Observe that $\mathbb{Q}_{h}$ is in fact a composition of locally defined $L^{2}$ projections into the polynomial space $P_{k+1}(T)$ for each element $T \in \mathcal{T}_{h}$.

In the usual finite element error analysis, one often reduces the error for finite element solutions into the error between the exact solution and an appropriately defined local projection or interpolation of the solution. For the WG-MFEM method discussed in previous sections, this refers to the error between the exact solution and its local $L^{2}$ projection. The difficulty in estimating the projection error arises from the fact that the finite element partition $\mathcal{T}_{h}$ contains arbitrary polygons or polyhedra that are different from the usual simplices as commonly employed in the standard finite element methods [14].

For simplicity of notation, we shall use $\lesssim$ to denote less than or equal to up to a constant independent of the mesh size, variables, or other parameters appearing in the inequality.

Lemma 4.1. Let $\mathcal{T}_{h}$ be a finite element partition of $\Omega$ satisfying the shape regularity assumptions A1-A4 as given in Section 3. Then, we have

$$
\begin{gathered}
\sum_{T \in \mathcal{T}_{h}}\left\|\mathbf{q}-Q_{0} \mathbf{q}\right\|_{T}^{2} \lesssim h^{2(s+1)}\|\mathbf{q}\|_{s+1}^{2}, \quad 0 \leq s \leq k, \\
\sum_{T \in \mathcal{T}_{h}}\left\|\nabla\left(\mathbf{q}-Q_{0} \mathbf{q}\right)\right\|_{T}^{2} \lesssim h^{2 s}\|\mathbf{q}\|_{s+1}^{2}, \quad 0 \leq s \leq k, \\
\sum_{T \in \mathcal{T}_{h}}\left(\left\|u-\mathbb{Q}_{h} u\right\|_{T}^{2}+h_{T}^{2}\left\|\nabla\left(u-\mathbb{Q}_{h} u\right)\right\|_{T}^{2}\right) \\
\lesssim h^{2(s+1)}\|u\|_{s+1}^{2}, \quad 0 \leq s \leq k+1 .
\end{gathered}
$$

Proof. To derive (4.1), let $S(T)$ be the circumscribed simplex of $T$ on which q can be defined by smooth extension if necessary. Let $\tilde{Q}_{0} \mathbf{q}$ be the projection of $\mathbf{q}$ in the element defined on $S(T)$. It follows that

$$
\left\|\mathbf{q}-Q_{0} \mathbf{q}\right\|_{T} \leq\left\|\mathbf{q}-\tilde{Q}_{0} \mathbf{q}\right\|_{T} \leq\left\|\mathbf{q}-\tilde{Q}_{0} \mathbf{q}\right\|_{S(T)} \lesssim h_{T}^{s+1}\|\mathbf{q}\|_{s+1, S(T)} .
$$

Using the above estimate we obtain

$$
\sum_{T \in \mathcal{T}_{h}}\left\|\mathbf{q}-Q_{0} \mathbf{q}\right\|_{T}^{2} \lesssim h^{2(s+1)} \sum_{T \in \mathcal{T}_{h}}\|\mathbf{q}\|_{s+1, S(T)}^{2} .
$$

It follows from the assumption $\mathbf{A} 4$ that the set of the circumscribed simplices $\left\{S(T): T \in \mathcal{T}_{h}\right\}$ has a fixed and small number of overlaps. Thus, the following estimate holds true:

$$
\sum_{T \in \mathcal{T}_{h}}\|\mathbf{q}\|_{s+1, S(T)}^{2} \lesssim\|\mathbf{q}\|_{s+1}^{2}
$$

Substituting the above inequality into (4.5) yields the desired estimate (4.1). 
To derive (4.2), we use the triangle inequality and the standard error estimate on $S(T)$ to obtain

$$
\begin{aligned}
\left\|\nabla\left(\mathbf{q}-Q_{0} \mathbf{q}\right)\right\|_{T} & \leq\left\|\nabla\left(\mathbf{q}-\tilde{Q}_{0} \mathbf{q}\right)\right\|_{S(T)}+\left\|\nabla\left(\tilde{Q}_{0} \mathbf{q}-Q_{0} \mathbf{q}\right)\right\|_{S(T)} \\
& \lesssim h_{T}^{s}\|\mathbf{q}\|_{s+1, S(T)}+h_{T}^{-1}\left\|\tilde{Q}_{0} \mathbf{q}-Q_{0} \mathbf{q}\right\|_{S(T)},
\end{aligned}
$$

where we have also used the standard inverse inequality in the second line. Notice that the assumption $\mathbf{A} \mathbf{3}$ on $\mathcal{T}_{h}$ implies that there exists a ball $B \subset T$ with a diameter proportional to $h_{T}$. Thus, we have from Lemma A.5 (see Appendix) that

$$
\begin{aligned}
\left\|\tilde{Q}_{0} \mathbf{q}-Q_{0} \mathbf{q}\right\|_{S(T)} & \lesssim\left\|\tilde{Q}_{0} \mathbf{q}-Q_{0} \mathbf{q}\right\|_{B} \leq\left\|\tilde{Q}_{0} \mathbf{q}-Q_{0} \mathbf{q}\right\|_{T} \\
& \leq\left\|\mathbf{q}-\tilde{Q}_{0} \mathbf{q}\right\|_{T}+\left\|\mathbf{q}-Q_{0} \mathbf{q}\right\|_{T} \\
& \lesssim h_{T}^{s+1}\|\mathbf{q}\|_{s+1, S(T)}
\end{aligned}
$$

Substituting the above estimate into (4.6) yields

$$
\left\|\nabla\left(\mathbf{q}-Q_{0} \mathbf{q}\right)\right\|_{T} \lesssim h_{T}^{s}\|\mathbf{q}\|_{s+1, S(T)} .
$$

Summing up the above estimate over $T \in \mathcal{T}_{h}$ leads to the desired estimate (4.2). Finally, the estimate (4.3) can be established analogously to (4.1) and (4.2).

We shall derive two equations that play useful roles in the error analysis for the WG-MFEM. The first equation is given by the following lemma.

Lemma 4.2. For any $\mathbf{q} \in\left[H^{1}(\Omega)\right]^{d}$, let $Q_{h} \mathbf{q} \in \mathcal{V}_{h}$ be the projection given by local $L^{2}$ projections. Then, on each element $T$, we have

$$
\left(\nabla_{w} \cdot\left(Q_{h} \mathbf{q}\right), w\right)_{T}=(\nabla \cdot \mathbf{q}, w)_{T}-\left\langle\mathbf{q} \cdot \mathbf{n}-Q_{b}(\mathbf{q} \cdot \mathbf{n}), w\right\rangle_{\partial T}
$$

for all $w \in P_{k+1}(T)$. Moreover, by summing (4.7) over all $T \in \mathcal{T}_{h}$ we obtain

$$
b\left(Q_{h} \mathbf{q}, w\right)=(\nabla \cdot \mathbf{q}, w)-\sum_{T \in \mathcal{T}_{h}}\left\langle\mathbf{q} \cdot \mathbf{n}-Q_{b}(\mathbf{q} \cdot \mathbf{n}), w\right\rangle_{\partial T} .
$$

Proof. For $\mathbf{q} \in\left[H^{1}(\Omega)\right]^{d}$, we apply the definition of $Q_{h}$ and the weak divergence $\nabla_{w} \cdot\left(Q_{h} \mathbf{q}\right)$ to obtain

$$
\begin{aligned}
\left(\nabla_{w} \cdot Q_{h} \mathbf{q}, w\right)_{T} & =-\left(Q_{0} \mathbf{q}, \nabla w\right)_{T}+\left\langle Q_{b}\left(\mathbf{q} \cdot \mathbf{n}_{e}\right) \mathbf{n}_{e} \cdot \mathbf{n}, w\right\rangle_{\partial T} \\
& =-\left(Q_{0} \mathbf{q}, \nabla w\right)_{T}+\left\langle Q_{b}(\mathbf{q} \cdot \mathbf{n}), w\right\rangle_{\partial T}
\end{aligned}
$$

for all $w \in P_{k+1}(T)$. Here we have used the fact that $\mathbf{n}_{e}= \pm \mathbf{n}$. Since $Q_{0}$ is the $L^{2}$ projection into $\left[P_{k}(T)\right]^{d}$, then

$$
\begin{aligned}
\left(Q_{0} \mathbf{q}, \nabla w\right)_{T} & =(\mathbf{q}, \nabla w)_{T} \\
& =-(\nabla \cdot \mathbf{q}, w)_{T}+\langle\mathbf{q} \cdot \mathbf{n}, w\rangle_{\partial T},
\end{aligned}
$$

where we have applied the usual divergence theorem in the second line. Substituting (4.10) into (4.9) yields (4.7). This completes the proof of the lemma.

The second equation is concerned with the bilinear form $\left(\nabla_{w} \cdot \mathbf{v}, \mathbb{Q}_{h} w\right)$ for $\mathbf{v} \in \mathcal{V}_{h}$ and $w \in H^{1}(\Omega)$. Using the definition of $\mathbb{Q}_{h}$ and the integration by parts, we have 
for any $\mathbf{v}=\left\{\mathbf{v}_{0}, \mathbf{v}_{b}\right\} \in \mathcal{V}_{h}$ and $w \in H^{1}(T)$ that

$$
\begin{aligned}
\left(\nabla_{w} \cdot \mathbf{v}, \mathbb{Q}_{h} w\right)_{T} & =-\left(\mathbf{v}_{0}, \nabla\left(\mathbb{Q}_{h} w\right)\right)_{T}+\left\langle\mathbf{v}_{b} \cdot \mathbf{n}, \mathbb{Q}_{h} w\right\rangle_{\partial T} \\
& =\left(\nabla \cdot \mathbf{v}_{0}, \mathbb{Q}_{h} w\right)_{T}+\left\langle\left(\mathbf{v}_{b}-\mathbf{v}_{0}\right) \cdot \mathbf{n}, \mathbb{Q}_{h} w\right\rangle_{\partial T} \\
& =\left(\nabla \cdot \mathbf{v}_{0}, w\right)_{T}+\left\langle\left(\mathbf{v}_{b}-\mathbf{v}_{0}\right) \cdot \mathbf{n}, \mathbb{Q}_{h} w\right\rangle_{\partial T} \\
& =-\left(\mathbf{v}_{0}, \nabla w\right)_{T}+\left\langle\mathbf{v}_{0} \cdot \mathbf{n}, w\right\rangle_{\partial T}+\left\langle\left(\mathbf{v}_{b}-\mathbf{v}_{0}\right) \cdot \mathbf{n}, \mathbb{Q}_{h} w\right\rangle_{\partial T} \\
& =-\left(\mathbf{v}_{0}, \nabla w\right)_{T}+\left\langle\left(\mathbf{v}_{0}-\mathbf{v}_{b}\right) \cdot \mathbf{n}, w-\mathbb{Q}_{h} w\right\rangle_{\partial T}+\left\langle\mathbf{v}_{b} \cdot \mathbf{n}, w\right\rangle_{\partial T} .
\end{aligned}
$$

The result can be summarized as follows.

Lemma 4.3. For any $\mathbf{v}=\left\{\mathbf{v}_{0}, \mathbf{v}_{b}\right\} \in \mathcal{V}_{h}$ and $w \in H^{1}(T)$ on the element $T \in \mathcal{T}_{h}$, one has

$$
\begin{aligned}
\left(\nabla_{w} \cdot \mathbf{v}, \mathbb{Q}_{h} w\right)_{T}= & -\left(\mathbf{v}_{0}, \nabla w\right)_{T}+\left\langle\left(\mathbf{v}_{0}-\mathbf{v}_{b}\right) \cdot \mathbf{n}, w-\mathbb{Q}_{h} w\right\rangle_{\partial T} \\
& +\left\langle\mathbf{v}_{b} \cdot \mathbf{n}, w\right\rangle_{\partial T} .
\end{aligned}
$$

Moreover, if $w \in H^{1}(\Omega)$, then the sum of (4.11) over all $T \in \mathcal{T}_{h}$ gives

$$
\begin{aligned}
b\left(\mathbf{v}, \mathbb{Q}_{h} w\right)= & -\left(\mathbf{v}_{0}, \nabla_{h} w\right)+\sum_{T \in \mathcal{T}_{h}}\left\langle\left(\mathbf{v}_{0}-\mathbf{v}_{b}\right) \cdot \mathbf{n}, w-\mathbb{Q}_{h} w\right\rangle_{\partial T} \\
& +\left\langle\mathbf{v}_{b} \cdot \mathbf{n}, w\right\rangle_{\partial \Omega},
\end{aligned}
$$

where $\nabla_{h} w$ is the gradient of $w$ taken element-by-element.

Let $T \in \mathcal{T}_{h}$ be any element, and $\mathbf{q} \in\left[H^{1}(T)\right]^{d}$. Then, we have from the definition of $Q_{b}$ that

$$
\begin{aligned}
\left\|\mathbf{q} \cdot \mathbf{n}-Q_{b}(\mathbf{q} \cdot \mathbf{n})\right\|_{\partial T}^{2} & =\left\langle\mathbf{q} \cdot \mathbf{n}-Q_{b}(\mathbf{q} \cdot \mathbf{n}), \mathbf{q} \cdot \mathbf{n}-Q_{b}(\mathbf{q} \cdot \mathbf{n})\right\rangle_{\partial T} \\
& \left.=\left\langle\mathbf{q} \cdot \mathbf{n}-Q_{b}(\mathbf{q} \cdot \mathbf{n}), \mathbf{q} \cdot \mathbf{n}-\left(Q_{0} \mathbf{q}\right) \cdot \mathbf{n}\right)\right\rangle_{\partial T} \\
& \leq\left\|\mathbf{q} \cdot \mathbf{n}-Q_{b}(\mathbf{q} \cdot \mathbf{n})\right\|_{\partial T}\left\|\mathbf{q} \cdot \mathbf{n}-\left(Q_{0} \mathbf{q}\right) \cdot \mathbf{n}\right\|_{\partial T}
\end{aligned}
$$

It follows that

$$
\left\|\mathbf{q} \cdot \mathbf{n}-Q_{b}(\mathbf{q} \cdot \mathbf{n})\right\|_{\partial T} \leq\left\|\mathbf{q} \cdot \mathbf{n}-\left(Q_{0} \mathbf{q}\right) \cdot \mathbf{n}\right\|_{\partial T} .
$$

Lemma 4.4. Let $\mathbf{q} \in\left[H^{k+1}(\Omega)\right]^{d}$ and $s$ be any real number such that $0 \leq s \leq k$. Then, we have

$$
\sum_{T \in \mathcal{T}_{h}} h_{T}\left\|\mathbf{q} \cdot \mathbf{n}-Q_{b}(\mathbf{q} \cdot \mathbf{n})\right\|_{\partial T}^{2} \lesssim h^{2(s+1)}\|\mathbf{q}\|_{s+1}^{2}
$$

and

$$
\left\|\mathbf{q}-Q_{h} \mathbf{q}\right\| \lesssim \rho h^{s+1}\|\mathbf{q}\|_{s+1} .
$$

Here $\|\cdot\|$ is a norm in $\mathcal{V}$ defined by (5.7)

Proof. Apply the trace inequality (A.18) (see Appendix) to the right-hand side of (4.13) to obtain

$$
h_{T}\left\|\mathbf{q} \cdot \mathbf{n}-Q_{b}(\mathbf{q} \cdot \mathbf{n})\right\|_{\partial T}^{2} \lesssim\left\|\mathbf{q}-Q_{0} \mathbf{q}\right\|_{T}^{2}+h_{T}^{2}\left\|\nabla\left(\mathbf{q}-Q_{0} \mathbf{q}\right)\right\|_{T}^{2} .
$$

Thus, it follows from Lemma 4.1 that the estimate (4.14) holds true. 
To derive (4.15), we have from the definition of the bilinear form $s(\cdot, \cdot)$ in (3.13), the definition of $Q_{b}$, and the inequality (4.13) that

$$
\begin{aligned}
s\left(\mathbf{q}-Q_{h} \mathbf{q}, \mathbf{q}-Q_{h} \mathbf{q}\right) & =\rho \sum_{T \in \mathcal{T}_{h}} h_{T}\left\|\left(\mathbf{q}-Q_{0} \mathbf{q}\right) \cdot \mathbf{n}-\left(\mathbf{q} \cdot \mathbf{n}-Q_{b}(\mathbf{q} \cdot \mathbf{n})\right)\right\|_{\partial T}^{2} \\
& \lesssim \rho \sum_{T \in \mathcal{T}_{h}} h_{T}\left(\left\|\left(\mathbf{q}-Q_{0} \mathbf{q}\right) \cdot \mathbf{n}\right\|_{\partial T}^{2}+\left\|\mathbf{q} \cdot \mathbf{n}-Q_{b}(\mathbf{q} \cdot \mathbf{n})\right\|_{\partial T}^{2}\right) \\
& \lesssim \rho \sum_{T \in \mathcal{T}_{h}} h_{T}\left\|\left(\mathbf{q}-Q_{0} \mathbf{q}\right) \cdot \mathbf{n}\right\|_{\partial T}^{2} .
\end{aligned}
$$

Now apply the trace inequality (A.18) to the last term of the above inequality to obtain

$$
s\left(\mathbf{q}-Q_{h} \mathbf{q}, \mathbf{q}-Q_{h} \mathbf{q}\right) \lesssim \rho \sum_{T \in \mathcal{T}_{h}}\left(\left\|\mathbf{q}-Q_{0} \mathbf{q}\right\|_{T}^{2}+h_{T}^{2}\left\|\nabla\left(\mathbf{q}-Q_{0} \mathbf{q}\right)\right\|_{T}^{2}\right) .
$$

Finally, it follows from the triple-bar norm definition (5.7) and the above inequality that

$$
\begin{aligned}
\left\|\mathbf{q}-Q_{h} \mathbf{q}\right\|^{2} & =a\left(\mathbf{q}-Q_{h} \mathbf{q}, \mathbf{q}-Q_{h} \mathbf{q}\right)+s\left(\mathbf{q}-Q_{h} \mathbf{q}, \mathbf{q}-Q_{h} \mathbf{q}\right) \\
& =\left\|\alpha^{\frac{1}{2}}\left(\mathbf{q}-Q_{0} \mathbf{q}\right)\right\|^{2}+s\left(\mathbf{q}-Q_{h} \mathbf{q}, \mathbf{q}-Q_{h} \mathbf{q}\right),
\end{aligned}
$$

which, combined with Lemma 4.1, gives rise to the desired estimate (4.15). This completes the proof.

\section{ERROR ANALYSIS}

The goal of this section is to derive some optimal order error estimates for the WG-MFEM approximation $\left(\mathbf{q}_{h} ; u_{h}\right)$ obtained from (3.14)-3.15). In finite element analysis, it is routine to decompose the error into two components in which the first is the difference between the exact solution and a properly defined projection of the exact solution while the second is the difference of the finite element solution with the same projection. In the current application, we shall employ the following decomposition:

$$
\begin{aligned}
\mathbf{q}-\mathbf{q}_{h} & =\left(\mathbf{q}-Q_{h} \mathbf{q}\right)+\left(Q_{h} \mathbf{q}-\mathbf{q}_{h}\right), \\
u-u_{h} & =\left(u-\mathbb{Q}_{h} u\right)+\left(\mathbb{Q}_{h} u-u_{h}\right) .
\end{aligned}
$$

For simplicity, we introduce the following notation:

$$
\mathbf{e}_{h}=\left\{\mathbf{e}_{0}, \mathbf{e}_{b}\right\}:=\mathbf{q}_{h}-Q_{h} \mathbf{q}, \quad \epsilon_{h}:=u_{h}-\mathbb{Q}_{h} u .
$$

Moreover, we shall denote $\sum_{T \in \mathcal{T}_{h}}\|\nabla w\|_{T}^{2}$ by $\left\|\nabla_{h} w\right\|^{2}$ for any $w \in \prod_{T \in \mathcal{T}_{h}} H^{1}(T)$.

5.1. Error equations. The goal here is to derive equations that $\mathbf{e}_{h}$ and $\epsilon_{h}$ must satisfy. To this end, for any finite element function $\mathbf{v}=\left\{\mathbf{v}_{0}, \mathbf{v}_{b}\right\} \in \mathcal{V}_{h}$, we test the first equation of (1.1) against $\mathbf{v}_{0}$ to obtain

$$
\left(\alpha \mathbf{q}, \mathbf{v}_{0}\right)+\left(\nabla u, \mathbf{v}_{0}\right)=0 .
$$

Now applying the identity (4.12) to the term $\left(\nabla u, \mathbf{v}_{0}\right)$ yields

$$
a(\mathbf{q}, \mathbf{v})-b\left(\mathbf{v}, \mathbb{Q}_{h} u\right)=\sum_{T \in \mathcal{T}_{h}}\left\langle\left(\mathbf{v}_{0}-\mathbf{v}_{b}\right) \cdot \mathbf{n}, \mathbb{Q}_{h} u-u\right\rangle_{\partial T}+\left\langle g, \mathbf{v}_{b} \cdot \mathbf{n}\right\rangle_{\partial \Omega},
$$


where the Dirichlet boundary condition (1.2) was also used. Recall that $a_{s}(\cdot, \cdot)=$ $a(\cdot, \cdot)+s(\cdot, \cdot)$. Adding and subtracting $a_{s}\left(Q_{h} \mathbf{q}, \mathbf{v}\right)$ in the equation above gives

$$
\begin{aligned}
a_{s}\left(Q_{h} \mathbf{q}, \mathbf{v}\right)-b\left(\mathbf{v}, \mathbb{Q}_{h} u\right)= & a\left(Q_{h} \mathbf{q}-\mathbf{q}, \mathbf{v}\right)+s\left(Q_{h} \mathbf{q}, \mathbf{v}\right)+\left\langle g, \mathbf{v}_{b} \cdot \mathbf{n}\right\rangle_{\partial \Omega} \\
& +\sum_{T \in \mathcal{T}_{h}}\left\langle\left(\mathbf{v}_{0}-\mathbf{v}_{b}\right) \cdot \mathbf{n}, \mathbb{Q}_{h} u-u\right\rangle_{\partial T} .
\end{aligned}
$$

It follows from the definition of the bilinear form $s(\cdot, \cdot)$ that, for any $\mathbf{w} \in\left[H^{1}(\Omega)\right]^{d}$, one has

$$
s(\mathbf{w}, \mathbf{v})=0, \quad \forall \mathbf{v} \in \mathcal{V} .
$$

Thus, we have $s\left(Q_{h} \mathbf{q}, \mathbf{v}\right)=s\left(Q_{h} \mathbf{q}-\mathbf{q}, \mathbf{v}\right)$ and

$$
a\left(Q_{h} \mathbf{q}-\mathbf{q}, \mathbf{v}\right)+s\left(Q_{h} \mathbf{q}, \mathbf{v}\right)=a_{s}\left(Q_{h} \mathbf{q}-\mathbf{q}, \mathbf{v}\right) .
$$

Substituting the above into (5.1) yields

$$
\begin{aligned}
a_{s}\left(Q_{h} \mathbf{q}, \mathbf{v}\right)-b\left(\mathbf{v}, \mathbb{Q}_{h} u\right)= & a_{s}\left(Q_{h} \mathbf{q}-\mathbf{q}, \mathbf{v}\right)+\left\langle g, \mathbf{v}_{b} \cdot \mathbf{n}\right\rangle_{\partial \Omega} \\
& +\sum_{T \in \mathcal{T}_{h}}\left\langle\left(\mathbf{v}_{0}-\mathbf{v}_{b}\right) \cdot \mathbf{n}, \mathbb{Q}_{h} u-u\right\rangle_{\partial T} .
\end{aligned}
$$

Next, we test the second equation of (1.1) against any $w \in \mathcal{W}_{h}$ to obtain

$$
(\nabla \cdot \mathbf{q}, w)=(f, w)
$$

Substituting (4.8) into the above equation yields

$$
b\left(Q_{h} \mathbf{q}, w\right)=(f, w)-\sum_{T \in \mathcal{T}_{h}}\left\langle\mathbf{q} \cdot \mathbf{n}-Q_{b}(\mathbf{q} \cdot \mathbf{n}), w\right\rangle_{\partial T} .
$$

Now, subtracting (5.3) from (3.14) gives

$$
\begin{aligned}
a_{s}\left(\mathbf{e}_{h}, \mathbf{v}\right)-b\left(\mathbf{v}, \epsilon_{h}\right) & =a_{s}\left(\mathbf{q}-Q_{h} \mathbf{q}, \mathbf{v}\right) \\
& -\sum_{T \in \mathcal{T}_{h}}\left\langle\left(\mathbf{v}_{0}-\mathbf{v}_{b}\right) \cdot \mathbf{n}, \mathbb{Q}_{h} u-u\right\rangle_{\partial T},
\end{aligned}
$$

for all $v \in \mathcal{V}_{h}$. Similarly, subtracting (5.4) from (3.15) yields

$$
b\left(\mathbf{e}_{h}, w\right)=\sum_{T \in \mathcal{T}_{h}}\left\langle\mathbf{q} \cdot \mathbf{n}-Q_{b}(\mathbf{q} \cdot \mathbf{n}), w\right\rangle_{\partial T},
$$

for all $w \in \mathcal{W}_{h}$.

The equations (5.5) and (5.6) constitute governing rules for the error term $\mathbf{e}_{h}$ and $\epsilon_{h}$. These are called error equations.

5.2. Boundedness and inf-sup condition. For any interior edge or flat face $e \in \mathcal{E}_{h}$, let $T_{1}$ and $T_{2}$ be two elements sharing $e$ in common. The jump of $w \in \mathcal{W}_{h}$ on $e$ is given by

$$
\llbracket w \rrbracket_{e}= \begin{cases}\left.w\right|_{\partial T_{1}}-\left.w\right|_{\partial T_{2}}, & e \in \mathcal{E}_{h}^{0} \\ w, & e \in \partial \Omega .\end{cases}
$$

We introduce a norm in $\mathcal{V}$ as follows:

$$
\|\mathbf{v}\|^{2}:=a(\mathbf{v}, \mathbf{v})+s(\mathbf{v}, \mathbf{v}), \quad \mathbf{v} \in \mathcal{V} .
$$

Furthermore, let $\|\cdot\|_{1, h}$ be a norm in the space $\mathcal{W}_{h}+H^{1}(\Omega)$ defined as follows:

$$
\|w\|_{1, h}^{2}:=\sum_{T \in \mathcal{T}_{h}}\|\nabla w\|_{T}^{2}+\sum_{e \in \mathcal{E}_{h}} h_{e}^{-1}\left\|Q_{b} \llbracket w \rrbracket\right\|_{e}^{2} .
$$


It is not hard to see that $\|\cdot\|_{1, h}$ defines a norm in $\mathcal{W}_{h}$ since $\|w\|_{1, h}=0$ would lead to $w=$ const on each element $T$ and $\llbracket w \rrbracket=Q_{b} \llbracket w \rrbracket=0$ on each edge or flat face $e \in \mathcal{E}_{h}$. It follows that $w=0$ on each element $T$. As to $\|\cdot\|$, assume that $\|\mathbf{v}\|=0$ for some $\mathbf{v} \in \mathcal{V}_{h}$; i.e.,

$$
\left(\alpha \mathbf{v}_{0}, \mathbf{v}_{0}\right)+\rho \sum_{T \in \mathcal{T}_{h}} h_{T}\left\langle\left(\mathbf{v}_{0}-\mathbf{v}_{b}\right) \cdot \mathbf{n},\left(\mathbf{v}_{0}-\mathbf{v}_{b}\right) \cdot \mathbf{n}\right\rangle_{\partial T}=0 .
$$

This implies that $\mathbf{v}_{0}=0$ on each element $T$ and $\left(\mathbf{v}_{0}-\mathbf{v}_{b}\right) \cdot \mathbf{n}=0$ on each edge or flat face $e \in \mathcal{E}_{h}$. Thus, we have $\mathbf{v}_{b} \cdot \mathbf{n}=0$ on each $e \in \mathcal{E}_{h}$. Recall that $\mathbf{v}_{b}$ is a vector parallel to $\mathbf{n}$ on each $e \in \mathcal{E}_{h}$. Hence, we must have $\mathbf{v}_{b}=0$ on each $e \in \mathcal{E}_{h}$. The other properties for a norm can be easily verified for $\|\cdot\|_{1, h}$ and $\|\cdot\|$.

It is not hard to see that the triple-bar norm is essentially a discrete $L^{2}$ norm, and the norm $\|\cdot\|_{1, h}$ is an $H^{1}$-equivalent norm in the corresponding space.

Lemma 5.1. For any $\varphi \in \mathcal{W}_{h}$, there exists at least one $\mathbf{v} \in \mathcal{V}_{h}$ such that

$$
\begin{aligned}
|b(\mathbf{v}, \varphi)| & =\|\varphi\|_{1, h}^{2}, \\
\|\mathbf{v}\| & \lesssim\|\varphi\|_{1, h} .
\end{aligned}
$$

Therefore, the following inf-sup condition is satisfied:

$$
\sup _{\mathbf{v} \in \mathcal{V}_{h},\|\mathbf{v}\| \neq 0} \frac{|b(\mathbf{v}, \varphi)|}{\|\mathbf{v}\|} \geq \beta\|\varphi\|_{1, h}
$$

for some positive constant $\beta$ independent of the meshsize $h$.

Proof. It follows from the definition of $b(\cdot, \cdot)$ and the discrete divergence that

$$
\begin{aligned}
b(\mathbf{v}, \varphi) & =\left(\nabla_{w} \cdot \mathbf{v}, \varphi\right) \\
& =\sum_{T \in \mathcal{T}_{h}}\left(\left\langle\mathbf{v}_{b} \cdot \mathbf{n}, \varphi\right\rangle_{\partial T}-\left(\mathbf{v}_{0}, \nabla \varphi\right)_{T}\right) \\
& =\sum_{e \in \mathcal{E}_{h}}\left\langle\mathbf{v}_{b} \cdot \mathbf{n}_{e}, \llbracket \varphi \rrbracket\right\rangle_{e}-\sum_{T \in \mathcal{T}_{h}}\left(\mathbf{v}_{0}, \nabla \varphi\right)_{T},
\end{aligned}
$$

provided that the jump $\llbracket \varphi \rrbracket$ was taken consistently with the direction set $\mathcal{D}_{h}$. Now by taking $\mathbf{v}_{0}=-\nabla \varphi$ on each element $T$ and $\mathbf{v}_{b}=h_{e}^{-1} Q_{b}(\llbracket \varphi \rrbracket) \mathbf{n}_{e}$ on each edge or flat face $e$ we arrive at

$$
b(\mathbf{v}, \varphi)=\sum_{T \in \mathcal{T}_{h}}(\nabla \varphi, \nabla \varphi)_{T}+\sum_{e \in \mathcal{E}_{h}} h_{e}^{-1}\left\langle Q_{b} \llbracket \varphi \rrbracket, Q_{b} \llbracket \varphi \rrbracket\right\rangle_{e}=\|\varphi\|_{1, h}^{2},
$$

which verifies (5.9) . To verify (5.10), we apply the definition of the triple-bar norm to the above chosen $\mathbf{v}$ to obtain

$$
\begin{aligned}
\|\mathbf{v}\|^{2} & =\left(\alpha \nabla_{h} \varphi, \nabla_{h} \varphi\right)+\rho \sum_{T \in \mathcal{T}_{h}} h_{T}\left\|\nabla \varphi \cdot \mathbf{n}+h_{e}^{-1} Q_{b}(\llbracket \varphi \rrbracket) \mathbf{n}_{e} \cdot \mathbf{n}\right\|_{\partial T}^{2} \\
& \lesssim\left\|\nabla_{h} \varphi\right\|^{2}+\sum_{e \in \mathcal{E}_{h}} h_{e}^{-1}\left\|Q_{b} \llbracket \varphi \rrbracket\right\|_{e}^{2}=\|\varphi\|_{1, h}^{2},
\end{aligned}
$$

which is what (5.10) states.

Lemma 5.2. The bilinear form $b(\cdot, \cdot)$ is bounded in $\mathcal{V}_{h} \times \mathcal{W}_{h}$. In other words, there exists a constant $C$ such that

$$
|b(\mathbf{v}, \varphi)| \leq C\|\mathbf{v}\|\|\varphi\|_{1, h} .
$$


Proof. We note from the definition of weak divergence that

$$
\begin{aligned}
b(\mathbf{v}, \varphi) & =\left(\nabla_{w} \cdot \mathbf{v}, \varphi\right) \\
& =\sum_{T \in \mathcal{T}_{h}}\left(\left\langle\mathbf{v}_{b} \cdot \mathbf{n}, \varphi\right\rangle_{\partial T}-\left(\mathbf{v}_{0}, \nabla \varphi\right)_{T}\right) \\
& =\sum_{e \in \mathcal{E}_{h}}\left\langle\mathbf{v}_{b} \cdot \mathbf{n}_{e}, \llbracket \varphi \rrbracket\right\rangle_{e}-\sum_{T \in \mathcal{T}_{h}}\left(\mathbf{v}_{0}, \nabla \varphi\right)_{T}
\end{aligned}
$$

Using Cauchy-Schwarz we obtain

$$
\begin{aligned}
|b(\mathbf{v}, \varphi)| & \leq \sum_{e \in \mathcal{E}_{h}}\left\|\mathbf{v}_{b} \cdot \mathbf{n}_{e}\right\|_{e}\|\llbracket \varphi \rrbracket\|_{e}+\sum_{T \in \mathcal{T}_{h}}\left\|\mathbf{v}_{0}\right\|_{T}\|\nabla \varphi\|_{T} \\
& \leq\left(\sum_{e \in \mathcal{E}_{h}} h_{e}\left\|\mathbf{v}_{b} \cdot \mathbf{n}_{e}\right\|_{e}^{2}\right)^{\frac{1}{2}}\left(\sum_{e \in \mathcal{E}_{h}} h_{e}^{-1}\|\llbracket \varphi \rrbracket\|_{e}^{2}\right)^{\frac{1}{2}}+\left\|\mathbf{v}_{0}\right\|\left\|\nabla_{h} \varphi\right\| \\
& \leq\left(\left(\sum_{e \in \mathcal{E}_{h}} h_{e}\left\|\mathbf{v}_{b} \cdot \mathbf{n}_{e}\right\|_{e}^{2}\right)^{\frac{1}{2}}+\left\|\mathbf{v}_{0}\right\|\right)\|\varphi\|_{1, h} .
\end{aligned}
$$

Let $T$ be an element that takes $e$ as an edge or flat face. Then, using the trace inequality (A.18) and the inverse inequality (A.38) we obtain

$$
\begin{aligned}
h_{e}\left\|\mathbf{v}_{b} \cdot \mathbf{n}_{e}\right\|_{e}^{2} & \leq 2 h_{e}\left\|\left(\mathbf{v}_{b}-\mathbf{v}_{0}\right) \cdot \mathbf{n}_{e}\right\|_{e}^{2}+2 h_{e}\left\|\mathbf{v}_{0} \cdot \mathbf{n}_{e}\right\|_{e}^{2} \\
& \lesssim h_{e}\left\|\left(\mathbf{v}_{b}-\mathbf{v}_{0}\right) \cdot \mathbf{n}_{e}\right\|_{e}^{2}+\left\|\mathbf{v}_{0}\right\|_{T}^{2}
\end{aligned}
$$

Substituting the above inequality into (5.13) yields

$$
|b(\mathbf{v}, \varphi)| \lesssim\|\mathbf{v}\|\|\varphi\|_{1, h}
$$

which completes the proof of the lemma.

5.3. Error estimates. We first establish some estimates useful in the forthcoming error estimates.

Lemma 5.3. Let $u \in H^{k+2}(\Omega)$ and $\mathbf{q} \in\left[H^{k+1}(\Omega)\right]^{d}$ be two smooth functions on $\Omega$. Then, the following estimates hold true:

$$
\begin{aligned}
& \sum_{T \in \mathcal{T}_{h}}\left|\left\langle\left(\mathbf{v}_{0}-\mathbf{v}_{b}\right) \cdot \mathbf{n}, \mathbb{Q}_{h} u-u\right\rangle_{\partial T}\right| \lesssim h^{s+1}\|u\|_{s+2}\|\mathbf{v}\|, \quad 0 \leq s \leq k \\
& \sum_{T \in \mathcal{T}_{h}}\left|\left\langle\mathbf{q} \cdot \mathbf{n}-Q_{b}(\mathbf{q} \cdot \mathbf{n}), \varphi\right\rangle_{\partial T}\right| \lesssim h^{s+1}\|\mathbf{q}\|_{s+1}\left\|\nabla_{h} \varphi\right\|, \quad 0 \leq s \leq k
\end{aligned}
$$

for all $\mathbf{v} \in \mathcal{V}$ and $\varphi \in \prod_{T \in \mathcal{T}_{h}} H^{1}(T)$ 
Proof. It follows from the Cauchy-Schwarz inequality, the definition of $\|\cdot\|$, and the trace inequality (A.18) that

$$
\begin{aligned}
\sum_{T \in \mathcal{T}_{h}} & \left|\left\langle\left(\mathbf{v}_{0}-\mathbf{v}_{b}\right) \cdot \mathbf{n}, \mathbb{Q}_{h} u-u\right\rangle_{\partial T}\right| \\
& \lesssim\left(\sum_{T \in \mathcal{T}_{h}} h_{T}\left\|\left(\mathbf{v}_{0}-\mathbf{v}_{b}\right) \cdot \mathbf{n}\right\|_{\partial T}^{2}\right)^{\frac{1}{2}} \cdot\left(\sum_{T \in \mathcal{T}_{h}} h_{T}^{-1}\left\|\mathbb{Q}_{h} u-u\right\|_{\partial T}^{2}\right)^{\frac{1}{2}} \\
& \lesssim\|\mathbf{v}\|\left(\sum_{T \in \mathcal{T}_{h}}\left(h_{T}^{-2}\left\|\mathbb{Q}_{h} u-u\right\|_{T}^{2}+\left\|\nabla\left(\mathbb{Q}_{h} u-u\right)\right\|_{T}^{2}\right)\right)^{\frac{1}{2}} \\
& \lesssim h^{s+1}\|\mathbf{v}\|\|u\|_{s+2},
\end{aligned}
$$

where we have used the estimate (4.3). This verifies the validity of (5.14).

Next, let $\bar{\varphi}$ be the average of $\varphi$ over each element $T$. It follows from the definition of $Q_{b}$, the estimates (4.14), and (A.18) that

$$
\begin{aligned}
& \sum_{T \in \mathcal{T}_{h}}\left|\left\langle\mathbf{q} \cdot \mathbf{n}-Q_{b}(\mathbf{q} \cdot \mathbf{n}), \varphi\right\rangle_{\partial T}\right|=\sum_{T \in \mathcal{T}_{h}}\left|\left\langle\mathbf{q} \cdot \mathbf{n}-Q_{b}(\mathbf{q} \cdot \mathbf{n}), \varphi-\bar{\varphi}\right\rangle_{\partial T}\right| \\
& \leq\left(\sum_{T \in \mathcal{T}_{h}} h_{T}\left\|\mathbf{q} \cdot \mathbf{n}-Q_{b}(\mathbf{q} \cdot \mathbf{n})\right\|_{\partial T}^{2}\right)^{\frac{1}{2}}\left(\sum_{T \in \mathcal{T}_{h}} h_{T}^{-1}\|\varphi-\bar{\varphi}\|_{\partial T}^{2}\right)^{\frac{1}{2}} \\
& \lesssim h^{s+1}\|\mathbf{q}\|_{s+1}\left(\sum_{T \in \mathcal{T}_{h}}\left(h_{T}^{-2}\|\varphi-\bar{\varphi}\|_{T}^{2}+\|\nabla \varphi\|_{T}^{2}\right)\right)^{\frac{1}{2}} \\
& \lesssim h^{s+1}\|\mathbf{q}\|_{s+1}\left\|\nabla_{h} \varphi\right\|,
\end{aligned}
$$

which completes the proof of (5.15).

With the help of Lemma 5.3, Lemma 5.1, and Lemma 5.2, we are now in a position to derive an error estimate for the WG-MFEM solution $\left(\mathbf{q}_{h} ; u_{h}\right)$ in the norm $\|\cdot\|$ and $\|\cdot\|_{1, h}$, respectively.

Theorem 5.4. Let $\mathbf{q}_{h} \in \mathcal{V}_{h}$ and $u_{h} \in \mathcal{W}_{h}$ be the solution of the weak Galerkin mixed finite element scheme (3.14)-(3.15). Assume that the exact solution $(\mathbf{q} ; u)$ of (1.3) -(1.4) is regular such that $u \in H^{s+2}(\Omega)$ and $\mathbf{q} \in\left[H^{s+1}(\Omega)\right]^{d}$ with $s \in(0, k]$. Then, one has

$$
\left\|\mathbf{q}_{h}-Q_{h} \mathbf{q}\right\|+\left\|u_{h}-\mathbb{Q}_{h} u\right\|_{1, h} \lesssim h^{s+1}\left(\|u\|_{s+2}+\|\mathbf{q}\|_{s+1}\right) .
$$

Proof. Let

$$
\ell(\mathbf{v}):=a_{s}\left(\mathbf{q}-Q_{h} \mathbf{q}, \mathbf{v}\right)-\sum_{T \in \mathcal{T}_{h}}\left\langle\left(\mathbf{v}_{0}-\mathbf{v}_{b}\right) \cdot \mathbf{n}, \mathbb{Q}_{h} u-u\right\rangle_{\partial T}
$$

be a linear functional on the finite element space $\mathcal{V}_{h}$. Also, let

$$
\chi(w):=\sum_{T \in \mathcal{T}_{h}}\left\langle\mathbf{q} \cdot \mathbf{n}-Q_{b}(\mathbf{q} \cdot \mathbf{n}), w\right\rangle_{\partial T}
$$

be a linear functional on the finite element space $\mathcal{W}_{h}$. The error equations (5.5) and (5.6) indicate that the pair $\left(\mathbf{e}_{h} ; \epsilon_{h}\right)$ is a solution of the following problem:

$$
\begin{aligned}
a_{s}\left(\mathbf{e}_{h}, \mathbf{v}\right)-b\left(\mathbf{v}, \epsilon_{h}\right) & =\ell(\mathbf{v}), \quad \forall \mathbf{v} \in \mathcal{V}_{h}, \\
b\left(\mathbf{e}_{h}, w\right) & =\chi(w), \quad \forall w \in \mathcal{W}_{h}
\end{aligned}
$$


The bilinear form $a_{s}(\cdot, \cdot)$ is clearly bounded, symmetric and positive definite in $\mathcal{V}_{h}$ equipped with the triple-bar norm $\|\cdot\|$. Lemma 5.2 indicates that the bilinear form $b(\cdot, \cdot)$ is bounded in $\mathcal{V}_{h} \times \mathcal{W}_{h}$, and Lemma 5.1 proved that the usual inf-sup condition of Babŭska $[3$ and Brezzi $[$ ] is satisfied. Thus, we have from the general theory of Babŭska and Brezzi that

$$
\left\|\mathbf{e}_{h}\right\|+\left\|\epsilon_{h}\right\|_{1, h} \leq C\left(\|\ell\|_{\mathcal{V}_{h}^{\prime}}+\|\chi\|_{\mathcal{W}_{h}^{\prime}}\right),
$$

where $\|\ell\|_{\mathcal{V}_{h}^{\prime}}$ and $\|\chi\|_{\mathcal{W}_{h}^{\prime}}$ are the norm of the corresponding linear functionals. To estimate the norms, we use (4.15) and (5.14) to come up with

$$
|\ell(\mathbf{v})| \lesssim h^{s+1}\left(\|\mathbf{q}\|_{s+1}+\|u\|_{s+2}\right)\|\mathbf{v}\| .
$$

Thus, we have

$$
\|\ell\|_{\mathcal{V}_{h}^{\prime}} \lesssim h^{s+1}\left(\|\mathbf{q}\|_{s+1}+\|u\|_{s+2}\right), \quad 0 \leq s \leq k .
$$

As to the norm of $\chi$, we use the estimate (5.15) to obtain

$$
|\chi(w)| \lesssim h^{s+1}\|\mathbf{q}\|_{s+1}\|w\|_{1, h}, \quad 0 \leq s \leq k,
$$

which implies that

$$
\|\chi\|_{\mathcal{W}_{h}^{\prime}} \lesssim h^{s+1}\|\mathbf{q}\|_{s+1}, \quad 0 \leq s \leq k .
$$

Substituting (5.20) and (5.21) into (5.19) yields the desired error estimate (5.16).

\section{ERRor ESTIMATES IN $L^{2}$}

To obtain an optimal order error estimate for the scalar component $\epsilon_{h}=u_{h}-\mathbb{Q}_{h} u$ in the usual $L^{2}$ norm, we consider a dual problem that seeks $\Psi$ and $\phi$ satisfying

$$
\begin{aligned}
\alpha \Psi+\nabla \phi & =0, \quad \text { in } \Omega, \\
\nabla \cdot \Psi & =\epsilon_{h}, \quad \text { in } \Omega, \\
\phi & =0, \quad \text { on } \partial \Omega .
\end{aligned}
$$

Assume that the usual $H^{2}$-regularity is satisfied for the dual problem; i.e., for any $\epsilon_{h} \in L^{2}(\Omega)$, there exists a unique solution $(\Psi ; \phi) \in\left[H^{1}(\Omega)\right]^{d} \times H^{2}(\Omega)$ such that

$$
\|\phi\|_{2}+\|\Psi\|_{1} \lesssim\left\|\epsilon_{h}\right\| .
$$

Lemma 6.1. Let $(\mathbf{q} ; u)$ be the solution of (1.3)-(1.4). Assume that $u \in H^{k+2}(\Omega)$, $\mathbf{q} \in\left[H^{k+1}(\Omega)\right]^{d}$, and the coefficient function satisfies $\alpha \in W^{1, \infty}(T)$ on each element T. Then, one has the following estimates:

$$
\begin{aligned}
\left|a_{s}\left(\mathbf{q}-Q_{h} \mathbf{q}, Q_{h} \Psi\right)\right| & \lesssim h^{k+2}\|\mathbf{q}\|_{k+1}\|\Psi\|_{1}, \\
\sum_{T \in \mathcal{T}_{h}}\left|\left\langle Q_{0} \Psi \cdot \mathbf{n}-Q_{b}(\Psi \cdot \mathbf{n}), \mathbb{Q}_{h} u-u\right\rangle_{\partial T}\right| & \lesssim h^{k+2}\|u\|_{k+2}\|\Psi\|_{1}, \\
\sum_{T \in \mathcal{T}_{h}}\left|\left\langle\mathbf{q} \cdot \mathbf{n}-Q_{b}(\mathbf{q} \cdot \mathbf{n}), \mathbb{Q}_{h} \phi-\phi\right\rangle_{\partial T}\right| & \lesssim h^{k+2}\|\mathbf{q}\|_{k+1}\|\phi\|_{2} .
\end{aligned}
$$


Proof. Let $\bar{\alpha}$ be the average of $\alpha$ on each element $T$. It follows from the definition of $a_{s}(\cdot, \cdot)$ and the approximation property (4.15) that

$$
\begin{aligned}
\left|a_{s}\left(\mathbf{q}-Q_{h} \mathbf{q}, Q_{h} \Psi\right)\right| & \leq\left|\left(\alpha\left(\mathbf{q}-Q_{0} \mathbf{q}\right), Q_{0} \Psi\right)\right|+\left|s\left(\mathbf{q}-Q_{0} \mathbf{q}, Q_{h} \Psi\right)\right| \\
& =\left|\left(\mathbf{q}-Q_{0} \mathbf{q},(\alpha-\bar{\alpha}) Q_{0} \Psi\right)\right|+\left|s\left(\mathbf{q}-Q_{0} \mathbf{q}, Q_{h} \Psi-\Psi\right)\right| \\
& \leq C h\|\nabla \alpha\|_{\infty}\left\|\mathbf{q}-Q_{0} \mathbf{q}\right\|\left\|Q_{0} \Psi\right\|+\left\|\mathbf{q}-Q_{h} \mathbf{q}\right\|\left\|\Psi-Q_{h} \Psi\right\| \\
& \lesssim h^{k+2}\|\mathbf{q}\|_{k+1}\|\Psi\|_{1},
\end{aligned}
$$

where $\|\nabla \alpha\|_{\infty}$ is the $L^{\infty}$ norm of $\nabla \alpha$ taken on each element. As to (6.6), we use (A.18) and (4.15) to obtain

$$
\begin{aligned}
& \sum_{T \in \mathcal{T}_{h}}\left|\left\langle Q_{0} \Psi \cdot \mathbf{n}-Q_{b}(\Psi \cdot \mathbf{n}), \mathbb{Q}_{h} u-u\right\rangle_{\partial T}\right| \\
& =\sum_{T \in \mathcal{T}_{h}}\left|\left\langle\left(Q_{0} \Psi-\Psi\right) \cdot \mathbf{n}-\left(Q_{b}(\Psi \cdot \mathbf{n})-\Psi \cdot \mathbf{n}\right), \mathbb{Q}_{h} u-u\right\rangle_{\partial T}\right| \\
& \quad \lesssim\left\|Q_{h} \Psi-\Psi\right\|\left(\sum_{T \in \mathcal{T}_{h}}\left(h_{T}^{-2}\left\|\mathbb{Q}_{h} u-u\right\|_{T}^{2}+\left\|\nabla\left(\mathbb{Q}_{h} u-u\right)\right\|_{T}^{2}\right)\right)^{1 / 2} \\
& \quad \lesssim h^{k+2}\|u\|_{k+2}\|\Psi\|_{1} .
\end{aligned}
$$

Similarly, using (4.14) we have

$$
\begin{aligned}
& \sum_{T \in \mathcal{T}_{h}}\left|\left\langle\mathbf{q} \cdot \mathbf{n}-Q_{b}(\mathbf{q} \cdot \mathbf{n}), \mathbb{Q}_{h} \phi-\phi\right\rangle_{\partial T}\right| \\
& \quad \lesssim h^{k+1}\|\mathbf{q}\|_{k+1}\left(\sum_{T \in \mathcal{T}_{h}}\left(h_{T}^{-2}\left\|\mathbb{Q}_{h} \phi-\phi\right\|_{T}^{2}+\left\|\nabla\left(\mathbb{Q}_{h} \phi-\phi\right)\right\|_{T}^{2}\right)\right)^{\frac{1}{2}} \\
& \quad \lesssim h^{k+2}\|\mathbf{q}\|_{k+1}\|\phi\|_{2} .
\end{aligned}
$$

This completes the proof of the lemma.

We are now in a position to establish an optimal order error estimate for the scalar/pressure component $\epsilon_{h}=u_{h}-\mathbb{Q}_{h} u$ in the usual $L^{2}$-norm. The result is stated in the following theorem.

Theorem 6.2. Let $\mathbf{q}_{h} \in \mathcal{V}_{h}$ and $u_{h} \in \mathcal{W}_{h}$ be the solution of (3.14)-(3.15). Assume that the exact solution of (1.3) -(1.4) satisfies $u \in H^{k+2}(\Omega)$ and $\mathbf{q} \in\left[H^{k+1}(\Omega)\right]^{d}$. Then, one has the following error estimate:

$$
\left\|u_{h}-\mathbb{Q}_{h} u\right\| \lesssim h^{k+2}\left(\|u\|_{k+2}+\|\mathbf{q}\|_{k+1}\right) .
$$

Proof. Testing (6.2) by $\epsilon_{h}=u_{h}-\mathbb{Q}_{h} u$ and then using (4.8) yields

$$
\begin{aligned}
\left(\epsilon_{h}, \epsilon_{h}\right) & =\left(\nabla \cdot \Psi, \epsilon_{h}\right) \\
& =b\left(Q_{h} \Psi, \epsilon_{h}\right)+\sum_{T \in \mathcal{T}_{h}}\left\langle\Psi \cdot \mathbf{n}-Q_{b}(\Psi \cdot \mathbf{n}), \epsilon_{h}\right\rangle_{\partial T} .
\end{aligned}
$$

It follows from (5.5) that

$$
\begin{aligned}
b\left(Q_{h} \Psi, \epsilon_{h}\right) & =a_{s}\left(\mathbf{e}_{h}, Q_{h} \Psi\right)-a_{s}\left(\mathbf{q}-Q_{h} \mathbf{q}, Q_{h} \Psi\right) \\
& +\sum_{T}\left\langle\left(Q_{0} \Psi\right) \cdot \mathbf{n}-Q_{b}(\Psi \cdot \mathbf{n}), \mathbb{Q}_{h} u-u\right\rangle_{\partial T} .
\end{aligned}
$$


Substituting the above equation into (6.9) yields

$$
\begin{aligned}
\left(\epsilon_{h}, \epsilon_{h}\right)= & a_{s}\left(\mathbf{e}_{h}, Q_{h} \Psi\right)-a_{s}\left(\mathbf{q}-Q_{h} \mathbf{q}, Q_{h} \Psi\right) \\
& +\sum_{T \in \mathcal{T}_{h}}\left\langle Q_{0} \Psi \cdot \mathbf{n}-Q_{b}(\Psi \cdot \mathbf{n}), \mathbb{Q}_{h} u-u\right\rangle_{\partial T} \\
& +\sum_{T \in \mathcal{T}_{h}}\left\langle\Psi \cdot \mathbf{n}-Q_{b}(\Psi \cdot \mathbf{n}), \epsilon_{h}\right\rangle_{\partial T} .
\end{aligned}
$$

Using (6.6) we obtain

$$
\sum_{T \in \mathcal{T}_{h}}\left\langle Q_{0} \Psi \cdot \mathbf{n}-Q_{b}(\Psi \cdot \mathbf{n}), \mathbb{Q}_{h} u-u\right\rangle_{\partial T} \lesssim h^{k+2}\|u\|_{k+2}\|\Psi\|_{1} .
$$

Using (5.15) we arrive at

$$
\sum_{T \in \mathcal{T}_{h}}\left\langle\Psi \cdot \mathbf{n}-Q_{b}(\Psi \cdot \mathbf{n}), \epsilon_{h}\right\rangle_{\partial T} \lesssim h\left\|\nabla_{h} \epsilon_{h}\right\|\|\Psi\|_{1}
$$

From 6.5) we have

$$
\left|a_{s}\left(\mathbf{q}-Q_{h} \mathbf{q}, Q_{h} \Psi\right)\right| \lesssim h^{k+2}\|\mathbf{q}\|_{k+1}\|\Psi\|_{1} .
$$

Now substituting (6.11)-(6.13) into (6.10) we obtain

(6.14) $\left(\epsilon_{h}, \epsilon_{h}\right) \lesssim\left|a_{s}\left(\mathbf{e}_{h}, Q_{h} \Psi\right)\right|+h^{k+2}\left(\|\mathbf{q}\|_{k+1}+\|u\|_{k+2}\right)\|\Psi\|_{1}+h\left\|\nabla_{h} \epsilon_{h}\right\|\|\Psi\|_{1}$.

It remains to deal with $\left|a_{s}\left(\mathbf{e}_{h}, Q_{h} \Psi\right)\right|$ in (6.14). To this end, we note that

$$
a_{s}\left(\mathbf{e}_{h}, Q_{h} \Psi\right)=a_{s}\left(\mathbf{e}_{h}, Q_{h} \Psi-\Psi\right)+a_{s}\left(\mathbf{e}_{h}, \Psi\right)
$$

and

$$
\left|a_{s}\left(\mathbf{e}_{h}, Q_{h} \Psi-\Psi\right)\right| \leq\left\|\mathbf{e}_{h}\right\|\left\|Q_{h} \Psi-\Psi\right\| \lesssim h\left\|\mathbf{e}_{h}\right\|\|\Psi\|_{1} .
$$

Thus,

$$
\left|a_{s}\left(\mathbf{e}_{h}, Q_{h} \Psi\right)\right| \lesssim\left|a_{s}\left(\mathbf{e}_{h}, \Psi\right)\right|+h\left\|\mathbf{e}_{h}\right\|\|\Psi\|_{1} .
$$

Since $\Psi \in\left[H^{1}(\Omega)\right]^{d}$, we have $a_{s}\left(\mathbf{e}_{h}, \Psi\right)=a\left(\mathbf{e}_{h}, \Psi\right)$; i.e., the stabilization term vanishes when one of the components is sufficiently regular. Now testing (6.1) against $\mathbf{e}_{h}$ gives

$$
a\left(\mathbf{e}_{h}, \Psi\right)=\left(\alpha \Psi, \mathbf{e}_{0}\right)=-\left(\nabla \phi, \mathbf{e}_{0}\right) .
$$

Furthermore, we have from (4.12) (with $\mathbf{v}=\mathbf{e}_{h}$ and $w=\phi$ ) and the fact that $\phi=0$ on $\partial \Omega$ that

$$
\left(\nabla \phi, \mathbf{e}_{0}\right)=-b\left(\mathbf{e}_{h}, \mathbb{Q}_{h} \phi\right)+\sum_{T \in \mathcal{T}_{h}}\left\langle\left(\mathbf{e}_{0}-\mathbf{e}_{b}\right) \cdot \mathbf{n}, \phi-\mathbb{Q}_{h} \phi\right\rangle_{\partial T} .
$$

Using the error equation (5.6) to replace the term $b\left(\mathbf{e}_{h}, \mathbb{Q}_{h} \phi\right)$ in above equation we obtain

$$
\begin{aligned}
\left(\nabla \phi, \mathbf{e}_{0}\right) & =-\sum_{T \in \mathcal{T}_{h}}\left\langle\mathbf{q} \cdot \mathbf{n}-Q_{b}(\mathbf{q} \cdot \mathbf{n}), \mathbb{Q}_{h} \phi\right\rangle_{\partial T}+\sum_{T \in \mathcal{T}_{h}}\left\langle\left(\mathbf{e}_{0}-\mathbf{e}_{b}\right) \cdot \mathbf{n}, \phi-\mathbb{Q}_{h} \phi\right\rangle_{\partial T} \\
& =\sum_{T \in \mathcal{T}_{h}}\left\langle\mathbf{q} \cdot \mathbf{n}-Q_{b}(\mathbf{q} \cdot \mathbf{n}), \phi-\mathbb{Q}_{h} \phi\right\rangle_{\partial T}+\sum_{T \in \mathcal{T}_{h}}\left\langle\left(\mathbf{e}_{0}-\mathbf{e}_{b}\right) \cdot \mathbf{n}, \phi-\mathbb{Q}_{h} \phi\right\rangle_{\partial T},
\end{aligned}
$$

where we have used the fact that $\phi \in H^{1}(\Omega)$ and $\phi=0$ on $\partial \Omega$ in the second line. Now using (6.7) to estimate the first summation in the above equation, and (5.14) with $s=0, \mathbf{v}=\mathbf{e}_{h}, u=\phi$ to estimate the second summation we obtain

$$
\left|\left(\nabla \phi, \mathbf{e}_{0}\right)\right| \lesssim\left(h^{k+2}\|\mathbf{q}\|_{k+1}+h\left\|\mathbf{e}_{h}\right\|\right)\|\phi\|_{2} .
$$


Thus,

$$
\left|a_{s}\left(\mathbf{e}_{h}, \Psi\right)\right|=\left|a\left(\mathbf{e}_{h}, \Psi\right)\right|=\left|\left(\nabla \phi, \mathbf{e}_{0}\right)\right| \lesssim\left(h^{k+2}\|\mathbf{q}\|_{k+1}+h\left\|\mathbf{e}_{h}\right\|\right)\|\phi\|_{2} .
$$

Substituting the above into (6.15) yields

$$
\left|a_{s}\left(\mathbf{e}_{h}, Q_{h} \Psi\right)\right| \lesssim\left(h^{k+2}\|\mathbf{q}\|_{k+1}+h\left\|\mathbf{e}_{h}\right\|\right)\|\phi\|_{2}+h\left\|\mathbf{e}_{h}\right\|\|\Psi\|_{1} .
$$

Now substituting (6.16) into (6.14) gives

$$
\begin{aligned}
\left(\epsilon_{h}, \epsilon_{h}\right) \lesssim & \left(h^{k+2}\|\mathbf{q}\|_{k+1}+h\left\|\mathbf{e}_{h}\right\|\right)\|\phi\|_{2}+h\left\|\mathbf{e}_{h}\right\|\|\Psi\|_{1} \\
& +h^{k+2}\left(\|\mathbf{q}\|_{k+1}+\|u\|_{k+2}\right)\|\Psi\|_{1}+h\left\|\nabla_{h} \epsilon_{h}\right\|\|\Psi\|_{1} .
\end{aligned}
$$

Observe that the $H^{2}$-regularity of the dual problem implies the following estimate

$$
\|\phi\|_{2}+\|\Psi\|_{1} \lesssim\left\|\epsilon_{h}\right\| .
$$

Substituting the above into (6.17) and then dividing both sides by $\left\|\epsilon_{h}\right\|$ we obtain

$$
\left\|\epsilon_{h}\right\| \lesssim h\left(\left\|\mathbf{e}_{h}\right\|+\left\|\nabla_{h} \epsilon_{h}\right\|\right)+h^{k+2}\left(\|\mathbf{q}\|_{k+1}+\|u\|_{k+2}\right),
$$

which, together with (5.16), implies the desired $L^{2}$ error estimate (6.8). This completes the proof.

\section{APPENDIX}

The goal of this appendix is to establish some fundamental estimates useful in the error estimate for WG-MFEM. First, we derive a trace inequality for functions defined on the finite element partition $\mathcal{T}_{h}$ with properties specified in Section 3 ,

Lemma A.3 (Trace Inequality). Let $\mathcal{T}_{h}$ be a partition of the domain $\Omega$ into polygons in $2 D$ or polyhedra in 3D. Assume that the partition $\mathcal{T}_{h}$ satisfies the assumptions (A1), (A2), and (A3) as specified in Section 3. Then, there exists a constant $C$ such that for any $T \in \mathcal{T}_{h}$ and edge/face $e \in \partial T$, we have

$$
\|\theta\|_{e}^{2} \leq C\left(h_{T}^{-1}\|\theta\|_{T}^{2}+h_{T}\|\nabla \theta\|_{T}^{2}\right),
$$

where $\theta \in H^{1}(T)$ is any function.

Proof. We shall provide a proof for the case of $e$ being a flat face and $\theta \in C^{1}(T)$. To this end, let the flat face $e$ be represented by the following parametric equation:

$$
\mathbf{x}_{e}=\phi(\xi, \eta):=\left(\phi_{1}(\xi, \eta), \phi_{2}(\xi, \eta), \phi_{3}(\xi, \eta)\right)
$$

for $(\xi, \eta) \in D \subset \mathbb{R}^{2}$. By Assumption A3, there exists a pyramid $P\left(e, T, A_{e}\right)$ with apex $A_{e}=\mathbf{x}_{*}:=\left(x_{1}^{*}, x_{2}^{*}, x_{3}^{*}\right)$ contained in the element $T$; see Figure1. This pyramid has the following parametric representation:

$$
\mathbf{x}(t, \xi, \eta)=(1-t) \phi(\xi, \eta)+t \mathbf{x}_{*}
$$

for $(t, \xi, \eta) \in[0,1] \times D$. For any given $\mathbf{x}_{e} \in e$, the line segment joining $\mathbf{x}_{e}$ and the apex $\mathbf{x}_{*}$ can be represented by

$$
\mathbf{x}(t)=\mathbf{x}_{e}+t\left(\mathbf{x}_{*}-\mathbf{x}_{e}\right) .
$$

From the fundamental theorem of Calculus, we have

$$
\theta^{2}\left(\mathbf{x}_{e}\right)-\theta^{2}(\mathbf{x}(t))=-\int_{0}^{t} \partial_{\tau} \theta^{2}\left(\mathbf{x}_{e}+\tau \omega\right) d \tau, \quad \omega=\mathbf{x}_{*}-\mathbf{x}_{e} .
$$


The above can be further rewritten as

$$
\theta^{2}\left(\mathbf{x}_{e}\right)-\theta^{2}(\mathbf{x}(t))=-2 \int_{0}^{t} \theta(\nabla \theta \cdot \omega) d \tau
$$

It follows from the Cauchy-Schwarz inequality that for any $t \in\left[0, \frac{1}{2}\right]$ we have

$$
\theta^{2}\left(\mathbf{x}_{e}\right) \leq \theta^{2}(\mathbf{x}(t))+h_{T}^{-1} \int_{0}^{\frac{1}{2}} \theta^{2}|\omega| d \tau+h_{T} \int_{0}^{\frac{1}{2}}|\nabla \theta|^{2}|\omega| d \tau
$$

Now we integrate the above inequality over the flat face $e$ by using the parametric equation (A.19), yielding

$$
\begin{aligned}
\int_{D} \theta^{2}\left(\mathbf{x}_{e}\right)\left|\phi_{\xi} \times \phi_{\eta}\right| d \xi d \eta & \leq \int_{D} \theta^{2}(\mathbf{x}(t))\left|\phi_{\xi} \times \phi_{\eta}\right| d \xi d \eta \\
& +h_{T}^{-1} \int_{0}^{\frac{1}{2}} \int_{D} \theta^{2}|\omega|\left|\phi_{\xi} \times \phi_{\eta}\right| d \xi d \eta d \tau \\
& +h_{T} \int_{0}^{\frac{1}{2}} \int_{D}|\nabla \theta|^{2}|\omega|\left|\phi_{\xi} \times \phi_{\eta}\right| d \xi d \eta d \tau
\end{aligned}
$$

Observe that the integral of a function over the prismatoid

$$
P_{\frac{1}{2}}:=\{\mathbf{x}(t, \xi, \eta): \quad(t, \xi, \eta) \in[0,1 / 2] \times D\}
$$

is given by

$$
\int_{P_{\frac{1}{2}}} f(\mathbf{x}) d \mathbf{x}=\int_{0}^{\frac{1}{2}} \int_{D} f(\mathbf{x}(\tau, \xi, \eta)) J(\tau, \xi, \eta) d \xi d \eta d \tau,
$$

where $J(\tau, \xi, \eta)=(1-\tau)^{2}\left|\left(\phi_{\xi} \times \phi_{\eta}\right) \cdot \omega\right|$ is the Jacobian from the coordinate change. The vector $\phi_{\xi} \times \phi_{\eta}$ is normal to the face $e$, and $\omega=\mathbf{x}_{*}-\mathbf{x}_{e}$ is a vector from the base point $\mathbf{x}_{e}$ to the apex $\mathbf{x}_{*}$. The angle assumption (see Assumption A3 of Section 3) for the prism $P\left(e, T, A_{e}\right)$ indicates that the Jacobian satisfies the relation

$$
J(\tau, \xi, \eta) \geq \frac{\mu_{0}}{4}\left|\phi_{\xi} \times \phi_{\eta}\right||\omega|, \quad \tau \in[0,1 / 2]
$$

for some fixed $\mu_{0} \in(0,1)$. Observe that the left-hand side of A.21 is the surface integral of $\theta^{2}$ over the face $e$. Thus, substituting (A.23) into (A.21) and (A.22) yields

$$
\begin{aligned}
\int_{e} \theta^{2} d e \leq & \int_{D} \theta^{2}(\mathbf{x}(t))\left|\phi_{\xi} \times \phi_{\eta}\right| d \xi d \eta \\
& +4 \mu_{0}^{-1} h_{T}^{-1} \int_{P_{\frac{1}{2}}} \theta^{2} d \mathbf{x}+4 \mu_{0}^{-1} h_{T} \int_{P_{\frac{1}{2}}}|\nabla \theta|^{2} d \mathbf{x} .
\end{aligned}
$$

Now we integrate the above with respect to $t$ in the interval $\left[0, \frac{1}{2}\right]$ to obtain

$$
\begin{aligned}
\frac{1}{2} \int_{e} \theta^{2} d e \leq & \int_{0}^{\frac{1}{2}} \int_{D} \theta^{2}(\mathbf{x}(t))\left|\phi_{\xi} \times \phi_{\eta}\right| d \xi d \eta d t \\
& +2 \mu_{0}^{-1} h_{T}^{-1} \int_{P_{\frac{1}{2}}} \theta^{2} d \mathbf{x}+2 \mu_{0}^{-1} h_{T} \int_{P_{\frac{1}{2}}}|\nabla \theta|^{2} d \mathbf{x} .
\end{aligned}
$$


Again, by substituting (A.23) into the right-hand side of (A.24) we arrive at

$$
\begin{aligned}
\frac{1}{2} \int_{e} \theta^{2} d e \leq & 4 \mu_{0}^{-1}|\omega|^{-1} \int_{0}^{\frac{1}{2}} \int_{D} \theta^{2}(\mathbf{x}(t)) J(t, \xi, \eta) d \xi d \eta d t \\
& +2 \mu_{0}^{-1} h_{T}^{-1} \int_{P_{\frac{1}{2}}} \theta^{2} d \mathbf{x}+2 \mu_{0}^{-1} h_{T} \int_{P_{\frac{1}{2}}}|\nabla \theta|^{2} d \mathbf{x} .
\end{aligned}
$$

The integral on the right-hand side of A.25 is the integral of $\theta^{2}$ on the prismatoid $P_{\frac{1}{2}}$. It can be seen from the Assumption A3 that

$$
|\omega|^{-1} \leq \alpha_{*} h_{T}^{-1}
$$

for some positive constant $\alpha_{*}$. Therefore, we have from the above estimate that

$$
\int_{e} \theta^{2} d e \leq C h_{T}^{-1} \int_{P_{\frac{1}{2}}} \theta^{2} d \mathbf{x}+C h_{T} \int_{P_{\frac{1}{2}}}|\nabla \theta|^{2} d \mathbf{x},
$$

which completes the proof of the lemma.

Next, we would like to establish an estimate for the $L^{2}$ norm of polynomial functions by their $L^{2}$ norm on a subdomain. To this end, we first derive a result of similar nature for general functions in $H^{1}$.

Lemma A.4. Let $K \subset \mathbb{R}^{d}$ be convex and $v \in H^{1}(K)$. Then,

$$
\|v\|_{K}^{2} \leq \frac{2|K|}{|S|}\|v\|_{S}^{2}+\frac{4 \delta^{2(d+1)}}{|S|^{2} d^{2}}\|\nabla v\|_{K}^{2},
$$

where $\delta$ is the diameter of $K$ and $S$ is any measurable subset of $K$.

Proof. Since $C^{1}(K)$ is dense in $H^{1}(K)$, it is sufficient to establish (A.26) for $v \in$ $C^{1}(K)$. For any $\mathbf{x}, \mathbf{y} \in K$, we have

$$
v^{2}(\mathbf{x})=v^{2}(\mathbf{y})-\int_{0}^{|\mathbf{x}-\mathbf{y}|} \partial_{r} v^{2}(\mathbf{x}+r \omega) d r, \quad \omega=\frac{\mathbf{y}-\mathbf{x}}{|\mathbf{y}-\mathbf{x}|} .
$$

From the usual chain rule and the Cauchy-Schwarz inequality we obtain

$$
\begin{aligned}
v^{2}(\mathbf{x}) & =v^{2}(\mathbf{y})-2 \int_{0}^{|\mathbf{x}-\mathbf{y}|} v \partial_{r} v(\mathbf{x}+r \omega) d r \\
& \leq v^{2}(\mathbf{y})+\epsilon \delta^{-1} \int_{0}^{|\mathbf{x}-\mathbf{y}|} v^{2} d r+\epsilon^{-1} \delta \int_{0}^{|\mathbf{x}-\mathbf{y}|}\left|\partial_{r} v\right|^{2} d r
\end{aligned}
$$

where $\epsilon>0$ is any constant. Let

$$
V(\mathbf{x})= \begin{cases}v^{2}(\mathbf{x}), & \mathbf{x} \in K \\ 0, & \mathbf{x} \notin K\end{cases}
$$

and

$$
W(\mathbf{x})= \begin{cases}\left|\partial_{r} v\right|^{2}(\mathbf{x}), & \mathbf{x} \in K, \\ 0, & \mathbf{x} \notin K .\end{cases}
$$

Then, the inequality (A.27) can be rewritten as

$$
v^{2}(\mathbf{x}) \leq v^{2}(\mathbf{y})+\epsilon \delta^{-1} \int_{0}^{\infty} V(\mathbf{x}+r \omega) d r+\epsilon^{-1} \delta \int_{0}^{\infty} W(\mathbf{x}+r \omega) d r .
$$


Integrating the above inequality with respect to $\mathbf{y}$ in $S$ yields

$$
\begin{aligned}
& |S| v^{2}(\mathbf{x}) \leq \int_{S} v^{2} d S \\
& +\int_{|\mathbf{x}-\mathbf{y}| \leq \delta}\left(\epsilon \delta^{-1} \int_{0}^{\infty} V(\mathbf{x}+r \omega) d r+\epsilon^{-1} \delta \int_{0}^{\infty} W(\mathbf{x}+r \omega) d r\right) d \mathbf{y} .
\end{aligned}
$$

It is not hard to see that

$$
\begin{aligned}
\int_{|\mathbf{x}-\mathbf{y}| \leq \delta} \int_{0}^{\infty} V(\mathbf{x}+r \omega) d r d \mathbf{y} & =\int_{0}^{\infty} \int_{|\omega|=1} \int_{0}^{\delta} V(\mathbf{x}+r \omega) \rho^{d-1} d \rho d \omega d r \\
& =\frac{\delta^{d}}{d} \int_{0}^{\infty} \int_{|\omega|=1} V(\mathbf{x}+r \omega) d \omega d r \\
& =\frac{\delta^{d}}{d} \int_{K}|\mathbf{x}-\mathbf{y}|^{1-d} v^{2}(\mathbf{y}) d \mathbf{y}
\end{aligned}
$$

Analogously, we have

$$
\int_{|\mathbf{x}-\mathbf{y}| \leq \delta} \int_{0}^{\infty} W(\mathbf{x}+r \omega) d r d \mathbf{y}=\frac{\delta^{d}}{d} \int_{K}|\mathbf{x}-\mathbf{y}|^{1-d}\left|\partial_{r} v(\mathbf{y})\right|^{2} d \mathbf{y} .
$$

Substituting (A.29) and (A.30) into (A.28) yields

$$
|S| v^{2}(\mathbf{x}) \leq \int_{S} v^{2} d S+\frac{\epsilon \delta^{d-1}}{d} \int_{K}|\mathbf{x}-\mathbf{y}|^{1-d} v^{2}(\mathbf{y}) d \mathbf{y}+\frac{\delta^{d+1}}{\epsilon d} \int_{K}|\mathbf{x}-\mathbf{y}|^{1-d}|\nabla v(\mathbf{y})|^{2} d \mathbf{y} .
$$

Now integrating both sides with respect to $\mathbf{x}$ in $K$ gives

$$
|S| \int_{K} v^{2} d K \leq|K| \int_{S} v^{2} d S+\frac{\epsilon \delta^{d}}{d} \int_{K} v^{2} d K+\frac{\delta^{d+2}}{\epsilon d} \int_{K}|\nabla v|^{2} d K
$$

which yields the desired estimate (A.26) by setting $\epsilon=\frac{|S| d}{2 \delta^{d}}$.

Consider the case of Lemma A.4 in which the convex domain $K$ is a shape regular $d$-simplex. Denote by $h_{K}$ the diameter of $K$. The shape regularity implies that

(1) the measure of $K$ is proportional to $h_{K}^{d}$,

(2) there exists an inscribed ball $B_{K} \subset K$ with diameter proportional to $h_{K}$.

Now let $S$ be a ball inside of $K$ with radius $r_{S} \geq \varsigma_{*} h_{K}$. Then, there exists a fixed constant $\kappa_{*}$ such that

$$
|K| \leq \kappa_{*}|S|
$$

Apply (A.31) in A.26) and notice that $\tau_{*}|S| \geq h_{K}^{d}$ and $\delta=h_{K}$. Thus,

$$
\|v\|_{K}^{2} \leq 2 \kappa_{*}\|v\|_{S}^{2}+\frac{4 \tau_{*}^{2} h_{K}^{2}}{d^{2}}\|\nabla v\|_{K}^{2} .
$$

For simplicity of notation, we shall rewrite A.32 in the following form:

$$
\|v\|_{K}^{2} \leq a_{0}\|v\|_{S}^{2}+a_{1} h_{K}^{2}\|\nabla v\|_{K}^{2} .
$$

If $v$ is infinitely smooth, then a recursive use of the estimate (A.33) yields the following result:

$$
\|v\|_{K}^{2} \leq \sum_{j=0}^{n} a_{j} h_{K}^{2 j}\left\|\nabla^{j} v\right\|_{S}^{2}+a_{n+1} h_{K}^{2 n+2}\left\|\nabla^{n+1} v\right\|_{K}^{2}
$$


In particular, if $v$ is a polynomial of degree $n$, then

$$
\|v\|_{K}^{2} \leq \sum_{j=0}^{n} a_{j} h_{K}^{2 j}\left\|\nabla^{j} v\right\|_{S}^{2} .
$$

The standard inverse inequality implies that

$$
\left\|\nabla^{j} v\right\|_{S} \lesssim h_{K}^{-j}\|v\|_{S} .
$$

Substituting the above into (A.35) gives

$$
\|v\|_{K}^{2} \lesssim\|v\|_{S}^{2} .
$$

The result is summarized as follows.

Lemma A.5 (Domain Inverse Inequality). Let $K \subset \mathbb{R}^{d}$ be a d-simplex which has diameter $h_{K}$ and is shape regular. Assume that $S$ is a ball in $K$ with diameter $r_{S}$ proportional to $h_{K}$; i.e., $r_{S} \geq \varsigma_{*} h_{K}$ with a fixed $\varsigma_{*}>0$. Then, there exists a constant $C=C\left(\varsigma_{*}, n\right)$ such that

$$
\|v\|_{K}^{2} \leq C\left(\varsigma_{*}, n\right)\|v\|_{S}^{2}
$$

for any polynomial $v$ of degree no more than $n$.

The usual inverse inequality in finite element analysis also holds true for piecewise polynomials defined on the finite element partition $\mathcal{T}_{h}$ provided that it satisfies the assumptions A1-A4 of Section 3.

Lemma A.6 (Inverse Inequality). Let $\mathcal{T}_{h}$ be a finite element partition of $\Omega$ consisting of polygons or polyhedra. Assume that $\mathcal{T}_{h}$ satisfies all the assumptions A1-A4 as specified in Section 3. Then, there exists a constant $C=C(n)$ such that

$$
\|\nabla \varphi\|_{T} \leq C(n) h_{T}^{-1}\|\varphi\|_{T}, \quad \forall T \in \mathcal{T}_{h}
$$

for any piecewise polynomial $\varphi$ of degree $n$ on $\mathcal{T}_{h}$.

Proof. The proof is merely a combination of Lemma A.5 and the standard inverse inequality on $d$-simplices. To this end, for any $T \in \mathcal{T}_{h}$, let $S(T)$ be the circumscribed simplex that is shape regular. It follows from the standard inverse inequality that

$$
\|\nabla \varphi\|_{T} \leq\|\nabla \varphi\|_{S(T)} \leq C h_{T}^{-1}\|\varphi\|_{S(T)} .
$$

Then we use the estimate (A.37), with $K=S(T)$, to obtain

$$
\|\nabla \varphi\|_{T} \leq C h_{T}^{-1}\|\varphi\|_{S} \leq C h_{T}^{-1}\|\varphi\|_{T}
$$

where $S$ is a ball inside of $T$ with a diameter proportional to $h_{T}$. This completes the proof of the lemma.

\section{REFERENCES}

[1] D. N. Arnold and F. Brezzi, Mixed and nonconforming finite element methods: implementation, postprocessing and error estimates (English, with French summary), RAIRO Modél. Math. Anal. Numér. 19 (1985), no. 1, 7-32. MR813687 (87g:65126)

[2] Douglas N. Arnold, Franco Brezzi, Bernardo Cockburn, and L. Donatella Marini, Unified analysis of discontinuous Galerkin methods for elliptic problems, SIAM J. Numer. Anal. 39 (2001/02), no. 5, 1749-1779, DOI 10.1137/S0036142901384162. MR.1885715(2002k:65183)

[3] Ivo Babuška, The finite element method with Lagrangian multipliers, Numer. Math. 20 (1972/73), 179-192. MR0359352 (50 \#11806)

[4] L. Beirão da Veiga, K. Lipnikov, and G. Manzini, Arbitrary-order nodal mimetic discretizations of elliptic problems on polygonal meshes, SIAM J. Numer. Anal. 49 (2011), no. 5, 1737-1760, DOI 10.1137/100807764. MR2837482(2012i:65229) 
[5] L. Beirão da Veiga, K. Lipnikov, and G. Manzini, Convergence analysis of the highorder mimetic finite difference method, Numer. Math. 113 (2009), no. 3, 325-356, DOI 10.1007/s00211-009-0234-6. MR2534128 (2010g:65180)

[6] M. Berndt, K. Lipnikov, D. Moulton, and M. Shashkov, Convergence of mimetic finite difference discretizations of the diffusion equation, East-West J. Numer. Math. 9 (2001), no. 4, 265-284. MR 1879474 (2003a:65085)

[7] Susanne C. Brenner and L. Ridgway Scott, The mathematical theory of finite element methods, Texts in Applied Mathematics, vol. 15, Springer-Verlag, New York, 1994. MR1278258 (95f:65001)

[8] F. Brezzi, On the existence, uniqueness and approximation of saddle-point problems arising from Lagrangian multipliers (English, with loose French summary), Rev. Française Automat. Informat. Recherche Opérationnelle Sér. Rouge 8 (1974), no. R-2, 129-151. MR0365287 (51 \#1540)

[9] Franco Brezzi and Michel Fortin, Mixed and hybrid finite element methods, Springer Series in Computational Mathematics, vol. 15, Springer-Verlag, New York, 1991. MR.1115205 (92d:65187)

[10] Franco Brezzi, Jim Douglas Jr., Ricardo Durán, and Michel Fortin, Mixed finite elements for second order elliptic problems in three variables, Numer. Math. 51 (1987), no. 2, 237-250, DOI 10.1007/BF01396752. MR890035 (88f:65190)

[11] Franco Brezzi, Jim Douglas Jr., and L. D. Marini, Two families of mixed finite elements for second order elliptic problems, Numer. Math. 47 (1985), no. 2, 217-235, DOI 10.1007/BF01389710. MR799685 (87g:65133)

[12] Franco Brezzi, Konstantin Lipnikov, and Mikhail Shashkov, Convergence of the mimetic finite difference method for diffusion problems on polyhedral meshes, SIAM J. Numer. Anal. 43 (2005), no. 5, 1872-1896 (electronic), DOI 10.1137/040613950. MR2192322 (2006j:65311)

[13] K. Chand and B. Henshaw, Overture Demo Introduction, 9th Overset Grid Symposium, Penn State University.

[14] Philippe G. Ciarlet, The finite element method for elliptic problems, North-Holland Publishing Co., Amsterdam, 1978. Studies in Mathematics and its Applications, Vol. 4. MR0520174 (58 \#25001)

[15] Bernardo Cockburn, Jayadeep Gopalakrishnan, and Raytcho Lazarov, Unified hybridization of discontinuous Galerkin, mixed, and continuous Galerkin methods for second order elliptic problems, SIAM J. Numer. Anal. 47 (2009), no. 2, 1319-1365, DOI 10.1137/070706616. MR2485455(2010b:65251)

[16] Daniele Antonio Di Pietro and Alexandre Ern, Mathematical aspects of discontinuous Galerkin methods, Mathématiques \& Applications (Berlin) [Mathematics \& Applications], vol. 69, Springer, Heidelberg, 2012. MR2882148

[17] Jérôme Droniou and Robert Eymard, A mixed finite volume scheme for anisotropic diffusion problems on any grid, Numer. Math. 105 (2006), no. 1, 35-71, DOI 10.1007/s00211-006-00341. MR2257385 (2008d:65121)

[18] Paola Causin and Riccardo Sacco, A discontinuous Petrov-Galerkin method with Lagrangian multipliers for second order elliptic problems, SIAM J. Numer. Anal. 43 (2005), no. 1, 280302 (electronic), DOI 10.1137/S0036142903427871. MR2177145 (2006h:65185)

[19] Jaroslav Fořt, Jiří Fürst, Jan Halama, Raphaèle Herbin, and Florence Hubert (eds.), Finite volumes for complex applications. VI. Problems \& perspectives. Volume 1, 2, Springer Proceedings in Mathematics, vol. 4, Springer, Heidelberg, 2011. MR2882736 (2012h:00040)

[20] Raphaèle Herbin and Florence Hubert, Benchmark on discretization schemes for anisotropic diffusion problems on general grids, Finite volumes for complex applications V, ISTE, London, 2008, pp. 659-692. MR2451465 (2010a:65211)

[21] A. Khamayseh1 and V. Almeida, Adaptive hybrid mesh refinement for multiphysics applications, Journal of Physics: Conference Series 78 (2007) 012039.

[22] L. Mu, J. Wang, and X. Ye, A weak Galerkin finite element methods with polynomial reduction, arXiv:1304.6481.

[23] L. Mu, J. Wang, and X. Ye, Weak Galerkin finite element methods on polytopal meshes, arXiv:1204.3655v2, submitted to International J. of Numerical Analysis and Modeling.

[24] L. Mu, J. Wang, Y. Wang, and X. Ye, A computational study of the weak Galerkin method for second order elliptic equations, Numerical Algorithms 63 (2013), 753-777. DOI:10.1007/s11075-012-9651-1. 
[25] L. Mu, J. Wang, X. Ye, and S. Zhao, A numerical study on the weak Galerkin method for the Helmholtz equation with large wave numbers, Communication in Computational Physics, accepted.

[26] L. Mu, J. Wang, G. Wei, X. Ye, and S. Zhao, Weak Galerkin methods for second order elliptic interface problems, Journal of Computational Physics 250 (2013), 106-125. doi:10.1016/j.jcp.2013.04.042.

[27] P. Raviart and J. Thomas, A mixed finite element method for second order elliptic problems, Mathematical Aspects of the Finite Element Method, I. Galligani, E. Magenes, eds., Lectures Notes in Math. 606, Springer-Verlag, New York, 1977.

[28] J. Wang, Mixed finite element methods, Numerical Methods in Scientific and Engineering Computing, Eds: W. Cai, Z. Shi, C-W. Shu, and J. Xu, Academic Press.

[29] Junping Wang and Xiu Ye, A weak Galerkin finite element method for second order elliptic problems, J. Comput. Appl. Math. 241 (2013), 103-115, DOI 10.1016/j.cam.2012.10.003. MR2994424

[30] Junping Wang and Xiu Ye, A weak Galerkin finite element method for the Stokes equation, arXiv:1302.2707, 2013.

Division of Mathematical Sciences, National Science Foundation, Arlington, VirGINIA 22230

E-mail address: jwang@nsf.gov

Department of Mathematics, University of Arkansas at Little Rock, Little Rock, ARKANSAS 72204

E-mail address: xxye@ualr.edu 